\title{
RSHL: a Ranging and Speed Measurement Method by Jointing Hyperbolic Frequency Modulation and Linear Frequency Modulation
}

\section{Peng Liu}

Hangzhou Institute of Applied accoustics

Caixia Song ( $\square$ cassiesong@qau.edu.cn)

Qingdao Agriculture University: Qingdao Agricultural University https://orcid.org/0000-0003-3897-7629

\section{Research}

Keywords: Hyperbolic Frequency Modulation, Linear Frequency Modulation, Doppler Invariance, Distance Measurement, Speed Measurement

Posted Date: June 15th, 2021

DOI: https://doi.org/10.21203/rs.3.rs-599257/v1

License: (c) (i) This work is licensed under a Creative Commons Attribution 4.0 International License.

Read Full License 


\title{
RSHL: a Ranging and Speed Measurement Method by Jointing Hyperbolic Frequency Modulation and Linear Frequency Modulation
}

\author{
Peng Liu ${ }^{1}$ and Caixia Song ${ }^{2 *}$
}

\footnotetext{
${ }^{*}$ Correspondence:

cassiesong@qau.edu.cn

${ }^{2}$ College of Science and

Information, Qingdao Agricultural

University, Qingdao, China

Full list of author information is

available at the end of the article
}

\begin{abstract}
In the waveform design, the distance measurement and resolution are a pair of irreconcilable contradictions. Linear Frequency Modulation (LFM) can alleviate this contradiction. LFM is widely used in radar and sonar, however, its Doppler tolerance is not ideal. Hyperbolic Frequency Modulation (HFM) signal has a particularly strong tolerance towards Doppler frequency shift. However, when the unidirectionally modulated HFM signal is in distance measurement, the Doppler delay of the matched filtering output cannot be eliminated, and there is a ranging error. After the echo signal of the combined HFM+LFM signal is matched filtering, the Doppler-induced delay is the same but opposite in direction, and the delay is closely related to the frequency, bandwidth, and pulse width of the transmitted signal. By using the combined signals, the ranging error in the ranging of single modulated HFM signal or LFM signal can be eliminated. In this paper, a Ranging and Speed measurement method by jointing Hyperbolic frequency modulation and Linear frequency modulation (RSHL) is proposed, which employs the same frequency band of positive and negative frequency modulation signals for speed measurement and ranging. In RSHL method, the signal parameters of the combined LFM+HFM signal can be set independently and no longer depend on each other. Therefore, the pulse width and frequency band in the combined LFM+HFM signal can be controlled independently, which can optimize the transmission signal form, reduce the operation cost, improve the measurement accuracy, and make full use of frequency band resources or pulse width resources.
\end{abstract}

Keywords: Hyperbolic Frequency Modulation; Linear Frequency Modulation; Doppler Invariance; Distance Measurement; Speed Measurement.

\section{Introduction}

Before the occurrence of pulse compression, in the waveform design, the distance measurement and resolution are a pair of irreconcilable contradictions, which can only be compromised. The appearance of pulse compression can solve this problem. Moreover, pulse compression technology has been widely used in radar and sonar[1][2][3][4]. In a large number of pulse compression signals, the Linear Frequency Modulation (LFM) signal is particularly popular for good pulse compression performance. LFM contains the following advantages:

1) LFM is a Doppler-tolerant waveform, which can tolerate a certain degree of unknown shift in Doppler frequency [5]. 
2) LFM is an equal-amplitude signal, which is beneficial to improve the transmission efficiency of peak power limited systems [6];

3) A higher range resolution can be obtained by increasing the bandwidth of LFM;

4) In addition, the spectrum of LFM is flat in the frequency band [7], and the generation and processing techniques of LFM are relatively mature [8].

According to the above advantages, LFM has been widely used in radar and sonar [9][10][11]. However, a large Doppler frequency shift will cause LFM matched filter mismatch and attenuate the output peak after LFM matched filter [12].

The Hyperbolic Frequency Modulation (HFM) signal is insensitive to Doppler, which can make up for the shortcomings of LFM. The traditional method of speed measurement is, according to the radial velocity of the target relative to the sonar, to calculate the speed through a set of narrow-band filter banks [7]. However, the higher the accuracy of the speed measurement, the more the number of Doppler filter banks needed will be multiplied. Therefore, the cost of the traditional method is too high.

In this paper, a Ranging and Speed measurement method by jointing Hyperbolic frequency modulation and Linear frequency modulation (RSHL) is proposed, which employs the same frequency band of positive and negative frequency modulation signals for speed measurement and ranging. In RSHL method, the signal parameters of the combined LFM+HFM signal can be set independently and no longer depend on each other. Therefore, the pulse width and frequency band in the combined LFM+HFM signal can be controlled independently. The RSHL method can not only accurately measure the speed, but also greatly decrease the ranging error caused by Doppler frequency shift in unidirectional modulation HFM. On the other hand, the RSHL method relaxes the bandwidth and frequency requirements, optimize the transmission signal form, reduce the operation cost, improve the measurement accuracy, and make full use of frequency band resources or pulse width resources. The main contributions of this paper are threefold:

1) Due to the coupling between distance and velocity in the fuzzy graph function of Frequency Modulation (FM) signal, the ranging deviation exists. The RSHL method takes advantage of the different time delays under different frequency bands and different pulse widths of LFM and HFM, and solves the problem of ranging errors caused by time delay.

2) Since a single FM signal cannot obtain the accurate starting point of time and cannot calculate the delay, the LFM and HFM combined signals are employed to calculate the starting point of time, and then the problem of speed measurement can be solved.

3) The advantages of combined signals of LFM and HFM are fully utilized, which not only solves the problem of speed measurement, but also can accurately measure distance.

\section{Related works}

In phased array radar network system[13], a Joint Target Assignment and Resource Optimization (JTARO) strategy is proposed for the application of multitarget tracking. The key mechanism of proposed JTARO strategy is to employ the optimization technique to jointly optimize the target-to-radar assignment, revisit time control, bandwidth, and dwell time allocation subject to several resource 
constraints, while achieving better tracking accuracies of multiple targets and low probability of intercept (LPI) performance of phased array radar network. A auto paired super-resolution range and velocity estimation method is proposed by using the OFDM integrated radar and communications waveform in [14], which to obtain the range and velocity estimations of other vehicles in the intelligent transportation system. Liu et al.[15] design an integrated system combining multiple-input multiple-output radar with orthogonal frequency division multiplexing (OFDM) communication. In this system, each antenna transmits the integrated waveform with a nonoverlapping block sub-frequency band. In order to sufficiently exploit the entire system bandwidth and array aperture, a joint time and space processing approach is proposed, and hence the range and angle estimations with high resolution are obtained, whereas the range and angle are coupled. The performance of a join$\mathrm{t}$ radar-communications receiver performing target detection while simultaneously decoding a message from an in-band communications user is analysed[16]. In this paper, inner bounds on the performance of the joint radar-communications receiver are then formulated.

Whyland et al.[17] proposed that Doppler insensitive waveforms, should be used to modulate pulses or sub-pulses of energy for probing a determined environment so that when the modulated energy is transmitted and received, the received energy may be processed. The HFM waveform has an inherent Doppler-invariant property. Y. Doisy et al.[18] derived the expressions of Doppler tolerance, Doppler and delay accuracy, and delay-Doppler ambiguity in case of high bandwidth duration product signals. The replicas of Doppler estimation and target range were reduced. Finally, results were applied to low-frequency active sonar. C. Lee et al.[19] proposed an underwater acoustic communication with hyperbolic frequency modulated waveforms. The received signal was demodulated by matched filtering of received signal and one hyperbolic chirp pulse. Simulation was performed to evaluate the performance of the proposed method. Wang, Fangyong et al.[20] proposed a method to estimate the target velocity using a combination of two HFM signals. They found that a HFM with an increasing frequency sweep (positive HFM) and one with a decreasing frequency sweep (negative HFM) yield a different time. And a better Doppler estimation can be obtained by using a negative HFM signal followed by a positive HFM signal than the other way around. The method was applied to real data and performance was demonstrated via simulated data. J. J. Murray[21] introduced an extended matched filter for HFM waveforms in active sonar systems, along with an exact closed-form solution for the Doppler bias in time of arrival estimates when using this filter. This solution applied to both broadband and narrowband HFM signals. Recently, the hyperbolic-frequency modulated signal has been widely employed in sonar systems for moving targets due to its Doppler tolerance, while the precise velocity estimation becomes a great challenge under such conditions. Huang, Shuxia et al.[22] proposed an improved method based on the sliding window matching algorithm to improve the performance. The method controlled the energy of environmental noise and interference by focusing on the dominant target highlight, and applying a designed window which utilizes the Doppler characteristics of hyperbolic-frequency modulated signals. The results verified the influence of the multi-highlights in velocity estimation and indicated that the improved method 
Table 1 Summary of important symbols.

\begin{tabular}{c|l}
\hline Symbol & Definition \\
\hline$f_{h 0}$ & The starting frequency of the HFM signal. \\
$f_{h 1}$ & The ending frequency of the HFM signal. \\
$f_{l 0}$ & The starting frequency of the LFM signal. \\
$f_{l 1}$ & The ending frequency of the LFM signal. \\
$T_{h}$ & The pulse width of the HFM signal. \\
$T_{l}$ & The pulse width of the LFM signal. \\
$s_{h}(t)$ & The HFM signal changing over time. \\
$s_{l}(t)$ & The LFM signal changing over time. \\
$\varphi$ & The phase of the HFM signal. \\
$s_{r}(t)$ & The received echo signal changing over time. \\
$f_{r}(t)$ & The instantaneous frequency of the received echo signal. \\
$\eta$ & Doppler compression or expansion factor. \\
$\tau_{h}$ & The delay of HFM signal. \\
$\tau_{l}$ & The delay of LFM signal. \\
$\mu$ & The modulation degree of LFM signal. \\
$\tau$ & The delay in the fuzzy function of LFM. \\
$\varepsilon$ & Frequency shift in the fuzzy function of LFM. \\
$\chi(\tau, \varepsilon)$ & The fuzzy function of Pulse Continuous Wave (PCW). \\
$t_{1}$ & The time when the matched filtering maximum appears in HFM signal. \\
$t_{2}$ & The time when the matched filtering maximum appears in LFM signal. \\
$R$ & The distance between the sonar and the target. \\
$v$ & The speed of the target.
\end{tabular}

has more effective performance. M. Kim et al.[23] investigated the performance of HFM signals for timing synchronization in underwater acoustic communication systems. The synchronization performance of the proposed HFM was then evaluated numerically using the channel model constructed based on western sea of South Korea. Numerical analysis suggests the HFM design achieving good performance for timing synchronization in presence of Doppler scale. Jedel, Andrzej et al.[24] proposed a sounding signal, which was a combination of Pseudo-Random Sequences (PRS), and elementary signals of HFM type. The structure of this signal was aimed at minimizing measurement error. They presented the idea of a sounding signal of HFM+PRS type, and the results of computer simulations.

Diamant et al.[25] presented a method for Doppler-shift estimation based on comparing the arrival times of two chirp signals and approximating the relation between this time difference and the Doppler shift ratio. This analysis also provides an interesting insight about the resilience of chirp signals to Doppler shift. The simulation results demonstrate improvement compared to commonly used benchmark methods in terms of accuracy of the Doppler shift estimation at near-Nyquist baseband sampling rates. LFM signals have been widely used for target detection in active sonar systems due to their robustness to reverberation.

\section{Method}

For convenience, Table 1 summarizes the main symbols together with their significances in this paper.

\subsection{The design of positive and negative FM waveform}

\subsubsection{HFM time domain waveform}

Let $T_{h}, f_{h 0}, f_{h 1}$ and $s_{h}(t)$ denote the pulse width of the HFM signal, the starting frequency of the HFM signal, the ending frequency of the HFM signal and the HFM signal changing over time, respectively. Then $s_{h}(t)$ can be expressed as follows: 


$$
s_{h}(t)=\left\{\begin{array}{c}
e^{-j \varphi_{0} \ln \left(\frac{t^{\prime}-t}{t^{\prime}}\right)} \quad 0 \leq t \leq T_{h} \\
0 \quad \text { otherwise }
\end{array}\right.
$$

where $t^{\prime}=\frac{f_{h 1}}{f_{h 1}-f_{h 0}} T_{h}$ and $\varphi_{0}=2 \pi f_{h 0} t^{\prime}$

When $f_{h 1}>f_{h 0}$, the HFM signal is called positive HFM, denoted as $\mathrm{HFM}^{+}$. When $f_{h 0}>f_{h 1}$, the HFM signal is called inverse HFM, denoted as $\mathrm{HFM}^{-}$.

\subsubsection{LFM time domain waveform}

Let $T_{l}, f_{l 0}, f_{l 1}$ and $s_{l}(t)$ denote the pulse width of the LFM signal, the starting frequency of the LFM signal, the ending frequency of the LFM signal and the LFM signal changing over time, respectively. Then $s_{l}(t)$ can be expressed as:

$$
s_{l}(t)=\left\{\begin{array}{c}
e^{-j 2 \pi\left(f_{l 0} t+\frac{1}{2} \mu t^{2}\right)} \quad 0 \leq t \leq T_{l} \\
0 \quad \text { otherwise }
\end{array}\right.
$$

where $\mu=\frac{B}{T_{l}}, B=f_{l 1}-f_{l 0}$.

When $f_{l 1}>f_{l 0}$, then the LFM signal is called positive LFM, denoted as $\mathrm{LFM}^{+}$. When $f_{l 0}>f_{l 1}$, then the LFM signal is called inverse LFM, denoted as LFM ${ }^{-}$.

\subsection{Speed measurement principle}

The higher the frequency used by sonar, the more serious the seawater absorption will be, and the greater the propagation loss will be, which is not conducive to the remote target detection. On the other hand, the electromagnetic waves used by radar to detect submarines in seawater can only travel a few kilometers, and then the energy will be absorbed by the seawater. And thus, in this paper, the formula derivation is based on the baseband low-frequency signal, without any additional carrier frequency, and the bandwidth is within a few hundred $\mathrm{Hz}$, which belongs to Low Power Radio Frequency (LPRF). When the RSHL method is used for radar, just change the parameter values such as bandwidth, frequency band, speed of sound, to radar parameter values.

\subsubsection{The effect of the speed of the target on the echo of the HFM signal}

(1) The effect of the moving speed of the target on the spectrum of the HFM+ signal

The relative movement between the sonar and the target will cause the received signal to change, which is represented by the shift of the signal frequency, and it is called the Doppler frequency shift phenomenon [26]. Let $v$ represent the speed of the target. It can be seen from Figure 1, when the target moves toward the sonar system, $v$ is positive and the echo frequency increases. Otherwise $v$ is negative and the echo frequency decreases.

(2) The effect of the moving speed and the direction of the target on the matched filtering of the FM signal

The matched processing gain of $10 \log (\mathrm{BT})$ can be obtained by employing broadband FM signal and echo matched filtering. It can be seen from Figure 2, with the increase of the moving speed of the target, the main peak value of $\mathrm{HFM}^{+}$signal 


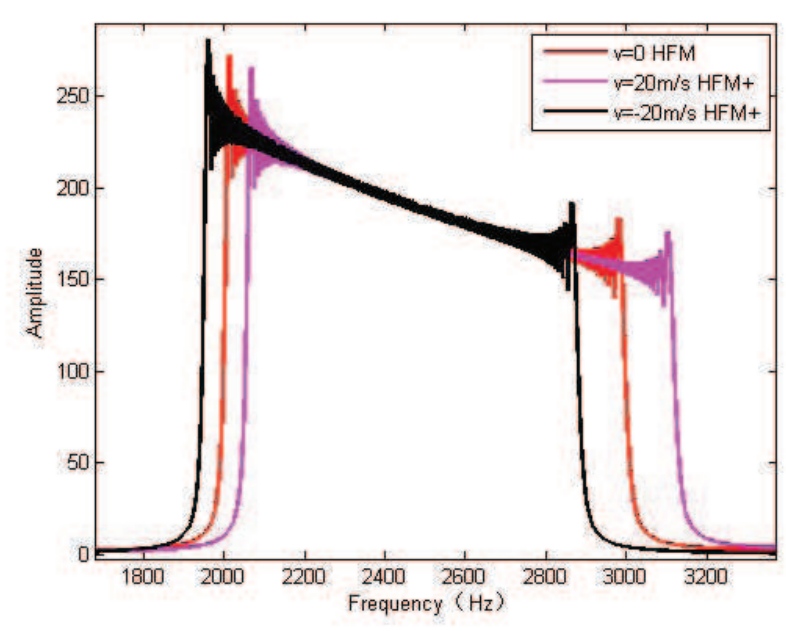

Figure 1 Effect of target moving speed on $\mathrm{HFM}^{+}$echo spectrum

matched filtering output decreases but does not expand [12]. When the direction of the target moving is different, the direction of delay caused by Doppler is also different.

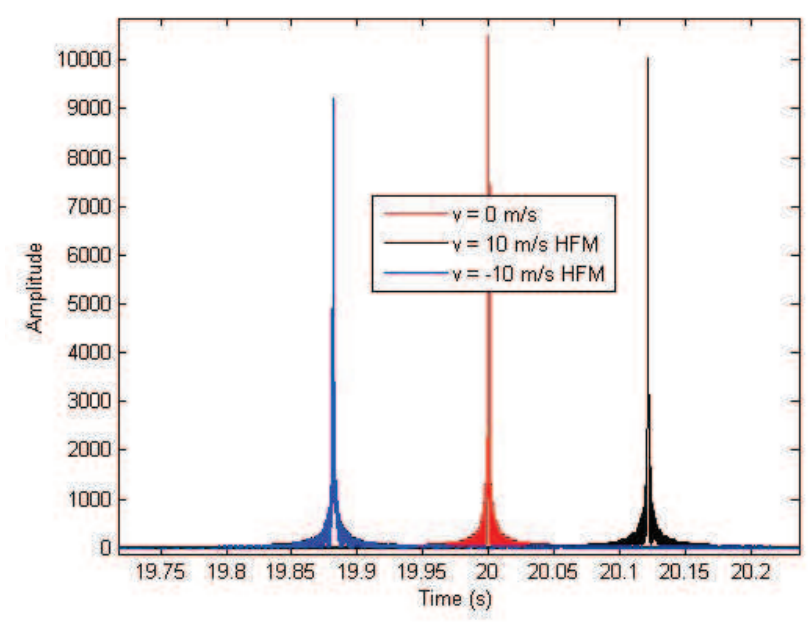

Figure 2 Influence of target speed magnitude and direction on $\mathrm{HFM}^{+}$signal matched filtering

3.2.2 The effect of the target speed on the LFM echo signal

(1) The effect of the moving speed of the target on the spectrum of the $L F M^{-}$signal

Figure 3 shows the effect of the target moving speed on $\mathrm{LFM}^{-}$echo spectrum. The effect of the moving speed of the target on the spectrum of $\mathrm{LFM}^{-}$signal is the same as that of the moving speed of the target on the spectrum of $\mathrm{HFM}^{+}$signal, which can be seen from Section 3.2.1-1.

(2) The effect of the moving speed and the direction of the target on the matched filtering of FM signal 


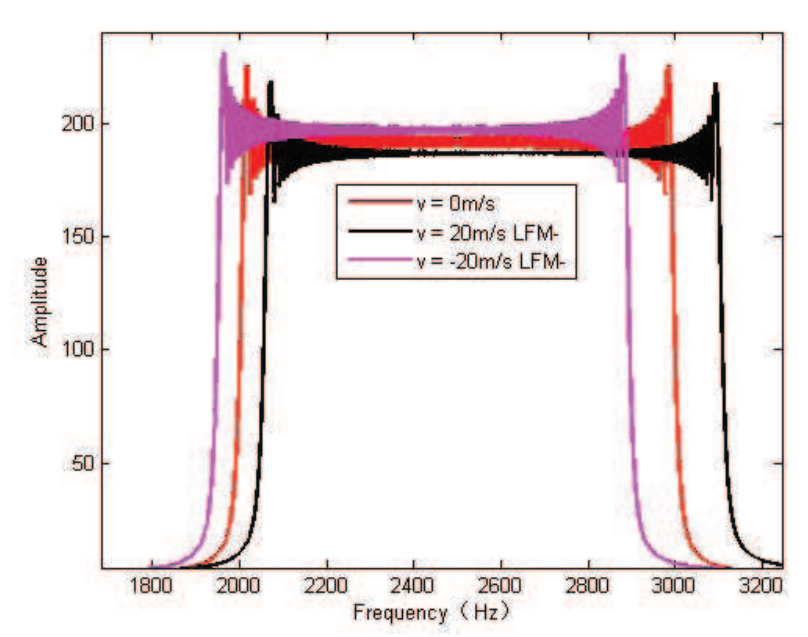

Figure 3 Influence of target moving speed on LFM- echo spectrum

Figure 4 shows the effect of target speed magnitude and direction on the matching filtering of $\mathrm{LFM}^{-}$signal. With the increase of the target speed, the main peak value of the matched filtering output of $\mathrm{LFM}^{-}$signal will not only decrease, but also be broadened [12]. When the movement direction of the target is different, the direction of delay caused by Doppler is also different.

Note: When the speed is positive, the leftmost time point is selected as the maximum point of the LFM matched filtering output. On the other hand, when the speed is negative, the rightmost time point is selected as the maximum point of the LFM matched filtering output.

\subsubsection{Doppler characteristic analysis of combined signals}

(1) Doppler invariance of $H F M$

According to Equation (1), the phase $\varphi$ of the HFM signal can be calculated by

$$
\varphi=2 \pi f_{h 0} \frac{f_{h 1}}{f_{h 1}-f_{h 0}} T_{h} \ln \left(\frac{\frac{f_{h 1}}{f_{h 1}-f_{h 0}} T_{h}-t}{\frac{f_{h 1}}{f_{h 1}-f_{h 0}} T_{h}}\right)
$$

Take the derivative with respect to $\varphi$, the instantaneous frequency $f_{s}(t)$ of HFM signal is

$$
\begin{aligned}
f_{s}(t) & =\frac{d \phi}{d t} \\
& =\frac{f_{h 0} f_{h 1}}{f_{h 1}-\left(f_{h 1}-f_{h 0}\right) \frac{t}{T_{h}}}
\end{aligned}
$$

When the target moves at speed $v$, the relative motion between the sonar and the target causes the transmitted signal with the pulse width $T_{h}$, at the receiving point, to become a signal with a pulse width $\frac{T_{h}}{\eta}$. Therefore, the pulse width of the echo is 


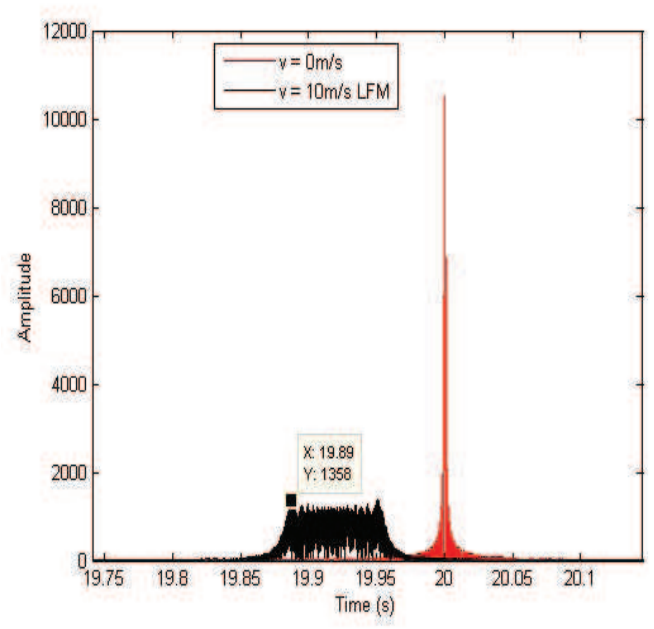

(a)

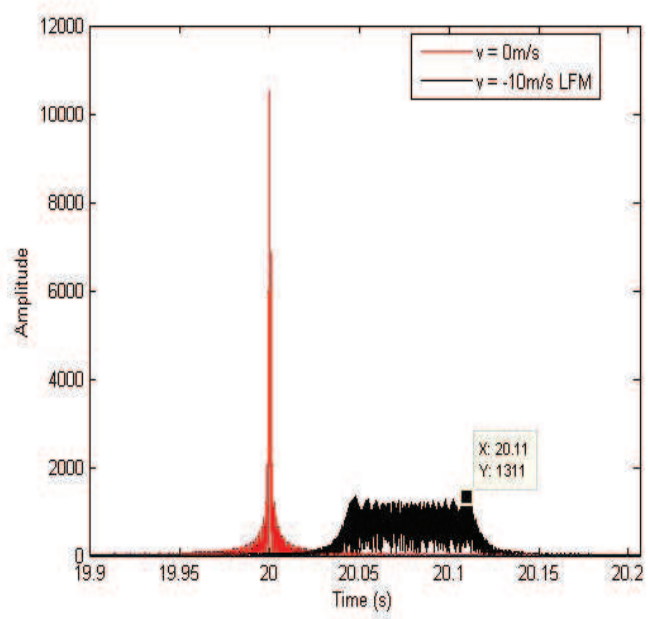

(b)

Figure 4 Effect of target speed magnitude and direction on the matching filtering of $\mathrm{LFM}^{-}$signal.

linearly compressed or stretched $\eta$ times [21], which can be seen from Figure 5. $\eta$ can be calculated by

$$
\eta=\frac{c+v}{c-v}
$$

where $c$ represents the speed of sound and its value is equal to $1500 \mathrm{~m} / \mathrm{s}$.

Then the received echo signal $s_{r}(t)$ can be calculated as:

$$
s_{r}(t)=\left\{\begin{array}{lc}
\left.e^{-j 2 \pi f_{h 0} \times \frac{f_{h 1}}{f_{h 1}-f_{h 0}} \times \frac{T_{h}}{\eta} \times \ln \left(\frac{\frac{f_{h 1}}{f_{h 1}-f_{h 0}} \times \frac{T_{h}}{\eta}-\eta t}{f_{h 1}}\right) \times \frac{T_{h}}{\eta}}\right) \\
0 \leq t \leq \frac{T_{h}}{\eta} \\
0 \quad \text { otherwise }
\end{array}\right.
$$


According to Equation (3) and Equation (4), the instantaneous frequency $f_{r}(t)$ of the received echo at this time can be calculated as:

$$
f_{r}(t)=\frac{f_{h 0} f_{h 1}}{\frac{f_{h 1}}{\eta}-\left(f_{h 1}-f_{h 0}\right) \frac{t}{T_{h}}}
$$

Since the HFM signal is insensitive to Doppler, the HFM signal has the characteristic of Doppler invariance [8]. Moreover, the change rule of the instantaneous frequency of the received signal remains unchanged, except that the instantaneous frequency $f_{s}(t)$ of the original signal is shifted by a time $\tau_{h}$, as shown in Figure 6 .

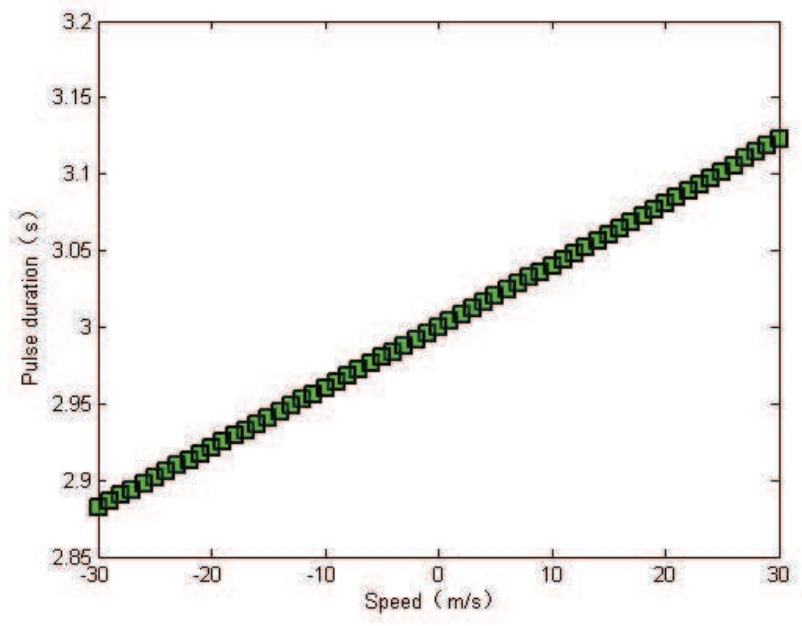

Figure 5 Influence of target speed on echo pulse width

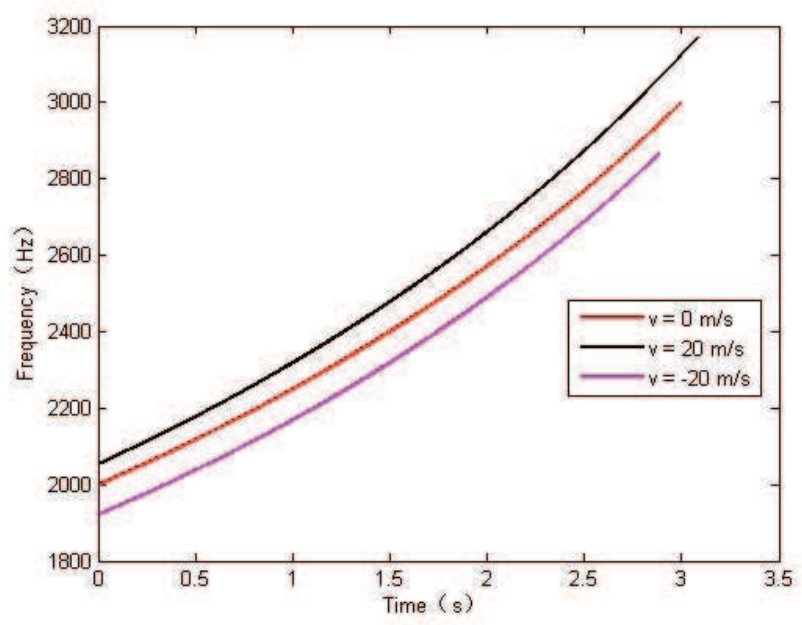

Figure 6 The effect of target speed on the $\mathrm{HFM}^{+}$echo spectrum 
Then we let

$$
f_{r}(t)=f_{s}\left(t-\tau_{h}\right)
$$

According to Equations (4) and (7), the matched filtering delay $\tau_{h}$ due to target Doppler can be obtained.

$$
\tau_{h}=\frac{f_{h 1}\left(\frac{1}{\eta}-1\right) T_{h}}{f_{h 1}-f_{h 0}}
$$

Because of $\mathrm{c} \gg v, \frac{1}{\eta}-1$ can be replaced by $\frac{-2 v}{c}$, and then $\tau_{h}$ can also be expressed as:

$$
\tau_{h}=\frac{f_{h 1} \times\left(-\frac{2 v}{c}\right) \times T_{h}}{f_{h 1}-f_{h 0}}
$$

(2) Doppler characteristics of LFM

Let $\chi(\tau, \varepsilon), \tau$ and $\varepsilon$ denote the fuzzy function of Pulse Continuous Wave (PCW), the delay in the fuzzy function $\chi(\tau, \varepsilon)$ and the frequency shift of LFM signal, respectively. We have

$$
\chi(\tau, \varepsilon)=\left\{\begin{array}{cc}
e^{j \pi \varepsilon\left(T_{l}-\tau\right) \frac{\sin \pi \varepsilon\left(T_{l}-|\tau|\right)}{\pi \varepsilon\left(T_{l}-|\tau|\right)}\left(\frac{T_{l}-|\tau|}{T_{l}}\right)} & |\tau|<T_{l} \\
0 \quad|\tau|>T_{l} &
\end{array}\right.
$$

According to the transformation property of the fuzzy function [27], the fuzzy function of LFM can be calculated:

$$
\chi(\tau, \varepsilon)=\left\{\begin{array}{cc}
e^{j\left(\pi(\varepsilon-\mu \tau)\left(T_{l}-\tau\right)-\mu \tau^{2} \pi\right) \frac{\sin \pi(\varepsilon-\mu \tau)\left(T_{l}-|\tau|\right)}{\pi(\varepsilon-\mu \tau)\left(T_{l}-\tau \mid\right)}\left(T_{l}-|\tau|\right)} \\
|\tau|<T_{l} \\
0 & |\tau|>T_{l}
\end{array}\right.
$$

where $\mu$ denotes the modulation degree of LFM signal. And then, we have

$$
|\chi(\tau, \varepsilon)|=\frac{\sin \pi(\varepsilon-\mu \tau)\left(T_{l}-|\tau|\right)}{\pi(\varepsilon-\mu \tau)\left(T_{l}-|\tau|\right)}\left(T_{l}-|\tau|\right)
$$

For $\varepsilon-\mu \tau=0$ section, we have

$$
\chi(\tau, \varepsilon)=T_{l}-|\tau|
$$

$\chi(\tau, \varepsilon)$ decreases linearly as $\tau$ increases, and then the pulse pressure delay caused by the moving target is

$$
\tau_{l}=\frac{\varepsilon}{\mu}
$$

When the sonar and the target are in relative motion, the received signal will have a frequency shift. Since LFM has certain Doppler tolerance, the instantaneous 
frequency of the received signal is only a delay. Therefore, a peak value can be obtained by using matched filtering, except that there is a delay $\tau_{l}$ at the peak position. $\tau_{l}$ can be calculated by

$$
\tau_{l}=\frac{2 v}{c} \times \frac{f_{l 0}+f_{l 1}}{2} \times \frac{T_{l}}{f_{l 1}-f_{l 0}}
$$

However, due to the delay time $\tau_{l}$ caused by Doppler, the distance of the target is determined by the time when the peak of the detector output appears. At this time, the measurement accuracy will be reduced, and there is a ranging error [26].

\subsubsection{Speed measurement principle}

Figure 7 shows the result of matched filter processing of the echo signal.

For the FM signal of an arbitrarily configured HFM+LFM combination signal, when the combined signals with the same modulation mode (both positive FM or both negative FM), the delay offset is proportional and the delay direction is the same. On the other hand, for combined signals with different modulations, the delay offset is proportional and the delay direction is opposite.

\subsection{Ranging and speed measurement based on arbitrary configuration of HFM+LFM combined signals}

Figure 8 shows the working process of the RSHL method. When active sonar work$\mathrm{s}$, the transmitting system transmits an acoustic signal with specific information into the seawater, which is called the transmitting signal. When the transmitting signal travels in seawater and meets targets, an echo signal will be generated. The echo signal propagates in the sea water according to the law of propagation, and reaches the hydrophone, which converts the acoustic signal into electrical signal. The electrical signal is processed by the signal (matching filtering, obtaining the arrival time of HFM and LFM, designing the function model and calculating) to obtain the distance and speed of the target.

Now let's derive the speed measurement formula, and we adopts the following assumptions:

a) It is assumed that the speed $v$ of the target is positive toward the sonar system.

b) A HFM signal is first transmitted, whose starting frequency, ending frequency and pulse width are $f_{h 0}, f_{h 1}$ and $T_{h}$, respectively. And then a LFM signal is transmitted, whose starting frequency, ending frequency and pulse width are $f_{l 0}, f_{l 1}$ and $T_{l}$, respectively.

Note: There is no size limit between $f_{h 0}$ and $f_{h 1}$, that is, both $f_{h 0}>=f_{h 1}$ and $f_{h 1}>=f_{h 0}$ are allowed. On the other hand, there is also no size limit between $f_{l 0}$ and $f_{l 1}$, that is, both $f_{l 0}>=f_{l 1}$ and $f_{l 1}>=f_{l 0}$ are allowed.

Let $t_{1}$ and $t_{2}$ represent the time when the matched filtering maximum appears in HFM and LFM, respectively. Let $R$ denotes the distance between the sonar and the target. We have

$$
t_{1}=\frac{2 R}{c}+\tau_{h}
$$




$$
t_{2}=\frac{2 R}{c}+\tau_{l}
$$

Based on Equation (17) and Equation (18), we have

$$
t_{1}-t_{2}=\tau_{h}-\tau_{l}
$$

Therefore, $v$ and $R$ can be calculated as Equation (20) and Equation (21), respectively. we have

$$
\begin{aligned}
& v=\frac{\frac{\left(t_{1}-t_{2}\right)}{2} c}{\frac{f_{h 1} T_{h}}{f_{h 1}-f_{h 0}}-\frac{\frac{f_{l 1}+f_{l 0}}{2} T_{l}}{f_{l 1}-f_{l 0}}} \\
& R=\frac{c}{2}\left(t_{1}+\frac{f_{h 1} T_{h}}{f_{h 1}-f_{h 0}} \times \frac{t_{1}-t_{2}}{\frac{\frac{f_{l 1}+f_{l 0}}{2} T_{l}}{f_{l 1}-f_{l 0}}-\frac{f_{h 1} T_{h}}{f_{h 1}-f_{h 0}}}\right)
\end{aligned}
$$

Equation (20) and (21) are the general form of $v$ and $R$, respectively. On the other hand, it can be seen that $T_{h}=T_{1}$ or $f_{h 1}=f_{l 1}$ or $f_{h 0}=f_{l 0}$ is special case of RSHL method.

Now we compute the time complexity of the proposed RSHL method. In RSHL method, after each single FM signal (HFM signal and LFM signal) is transmitted separately, the received echo signals and the transmitted signals are matched and filtered to obtain the target velocity and range information of the target. When the combined HFM and LFM signals are transmitted separately, the time complexity is $O(1)$. When the echo signals are matched and filtered, the copy signals are combined signals, the time complexity is $O(1)$. Based on the above analysis, the time complexity of RSHL method is $O(1)$.

In this section, the waveform design and implementation of combined LFM and HFM signals are introduced in detail. First of all, LFM signal and HFM signal are controlled separately, and the pulse width, frequency band and modulation mode of the two signals are set independently at will, and then the two signals are combined together to transmit. Then, the received echo signal and the transmitted signal are matched and filtered to detect the presence of the target. The method presented in this paper is versatile and easy to implement in engineering applications.

\section{Results and Discussion}

\subsection{Simulation Settings}

We set up four simulation environments for experimental comparison: Simulation Environment 1, Simulation Environment 2 and Simulation Environment 3.

Simulation Environment 1: In the HFM signal, the starting frequency, the ending frequency and pulse width are $f_{h 0}=2000 \mathrm{~Hz}, f_{h 1}=3000 \mathrm{~Hz}$ and $T_{h}=3 \mathrm{~s}$, respectively. In the LFM signal, the starting frequency, the ending frequency and pulse width are $f_{l 0}=1000 \mathrm{~Hz}, f_{l 1}=900 \mathrm{~Hz}$ and $T_{l}=4 \mathrm{~s}$, respectively. For both HFM signal and 
Table 2 Results under Simulation Environment 1

\begin{tabular}{|c|c|c|c|c|c|c|c|c|c|c|}
\hline Speed $v$ & \multicolumn{2}{|c|}{ Peak moment } & Speed measurement & Ranging of & Speed measurement & Ranging error & \multicolumn{2}{|c|}{$\begin{array}{l}\text { Ranging error of single signal } \\
\end{array}$} & \multicolumn{2}{|c|}{ Range accuracy improvement ratio } \\
\hline$(\mathrm{m} / \mathrm{s})$ & $t_{1}$ & $t_{2}$ & of RSHL $(\mathrm{m} / \mathrm{s})$ & $\mathrm{RSHL}(\mathrm{km})$ & error of RSHL & of RSHL & HFM & LFM & Compared to HFM & Compared to LFM \\
\hline 15 & 20.1834 & 19.2644 & 14.6649 & 15.0056 & $2.234 \%$ & $0.037333 \%$ & $0.917 \%$ & $3.678 \%$ & $95.92875 \%$ & $98.98496 \%$ \\
\hline 10 & 20.1216 & 19.4904 & 10.0714 & 15.0005 & $0.714 \%$ & $0.003333 \%$ & $0.608 \%$ & $2.548 \%$ & $99.45175 \%$ & $99.86918 \%$ \\
\hline 5 & 20.0604 & 19.7489 & 4.9719 & 15.0006 & $0.562 \%$ & $0.004 \%$ & $0.302 \%$ & $1.2555 \%$ & $98.6755 \%$ & $99.6814 \%$ \\
\hline-15 & 19.8237 & 20.7401 & -14.6239 & 14.9994 & $2.507333 \%$ & $0.004 \%$ & $0.8815 \%$ & $3.7005 \%$ & $99.54623 \%$ & $99.89191 \%$ \\
\hline-10 & 19.8816 & 20.4959 & -9.8024 & 14.9994 & $1.976 \%$ & $0.004 \%$ & $0.592 \%$ & $2.4795 \%$ & $99.32432 \%$ & $99.83868 \%$ \\
\hline-3 & 19.9643 & 20.1434 & -2.8587 & 14.9989 & $4.71 \%$ & $0.007333 \%$ & $0.1785 \%$ & $0.717 \%$ & $95.89169 \%$ & $98.97722 \%$ \\
\hline
\end{tabular}

LFM signal, the sample frequency $f_{s a}=7000 \mathrm{~Hz}$, Signal to Noise Ratio (SNR) is $-20 \mathrm{~dB}$ in the echo signal, and the distance between the target and the sound source is $15 \mathrm{~km}$.

Simulation Environment 2: In the HFM signal, the starting frequency, the ending frequency and pulse width are $f_{h 0}=2000 \mathrm{~Hz}, f_{h 1}=2200 \mathrm{~Hz}$ and $T_{h}=4 \mathrm{~s}$, respectively. In the LFM signal, the starting frequency, the ending frequency and pulse width are $f_{l 0}=1700 \mathrm{~Hz}, f_{l 1}=1100 \mathrm{~Hz}$ and $T_{l}=3 \mathrm{~s}$, respectively. For both HFM signal and LFM signal, the sample frequency $f_{s a}=7000 \mathrm{~Hz}, \mathrm{SNR}$ is $-20 \mathrm{~dB}$ in the echo signal, and the distance between the target and the sound source is $15 \mathrm{~km}$.

Simulation Environment 3: In the HFM signal, the starting frequency, the ending frequency and pulse width are $f_{h 0}=2000 \mathrm{~Hz}, f_{h 1}=3000 \mathrm{~Hz}$ and $T_{h}=7 \mathrm{~s}$, respectively. In the LFM signal, the starting frequency, the ending frequency and pulse width are $f_{l 0}=1000 \mathrm{~Hz}, f_{l 1}=1300 \mathrm{~Hz}$ and $T_{l}=3 \mathrm{~s}$, respectively. For both HFM signal and LFM signal, the sample frequency $f_{s a}=7000 \mathrm{~Hz}$, SNR is $-20 \mathrm{~dB}$ in the echo signal, and the distance between the target and the sound source is $15 \mathrm{~km}$.

Simulation Environment 4: In the HFM signal, the starting frequency, the ending frequency and pulse width are $f_{h 0}=2100 \mathrm{~Hz}, f_{h 1}=2200 \mathrm{~Hz}$ and $T_{h}=1 \mathrm{~s}$, respectively. In the LFM signal, the starting frequency, the ending frequency and pulse width are $f_{l 0}=2200 \mathrm{~Hz}, f_{l 1}=2100 \mathrm{~Hz}$ and $T_{l}=1 \mathrm{~s}$, respectively. For both HFM signal and LFM signal, the sample frequency $f_{s a}=7000 \mathrm{~Hz}$, SNR is $-16 \mathrm{~dB}$ in the echo signal, and the gain is $10 \log \left(100^{*} 1\right)-16=4 \mathrm{~dB}$, which is a low SNR. The distance between the target and the sound source is $15 \mathrm{~km}$.

\subsection{Comparison with Existing Studies}

Figure 9 shows the performance analysis under the simulation environment 1 under various SNRs. Table 2 gives the numerical results of RSHL, LFM and HFM.

We take Figure 9(a) as an example to analyze the performance of RSHL method under various SNRs. From Figure 9(a), it can be seen that, in the RSHL method, after matched filtering, the maximum points $t_{1}=20.1834$ and $t_{2}=19.2644$. Based on Equation (21), $R$ is equal to $15.0056 \mathrm{~km}$. According to Equation (20), the value of $v$ is $14.6649 \mathrm{~m} / \mathrm{s}$. The speed measurement and ranging error of RSHL are $2.234 \%$ and $0.03733 \%$, respectively. The ranging error of HFM and LFM are $0.917 \%$ and $3.678 \%$, respectively. It can be seen from Table 2 that, compared with LFM and HFM, the range measurement accuracy of RSHL is improved by $95.92875 \%$ and $98.98496 \%$, respectively.

Figure 10 shows the performance analysis under the simulation environment 1 under various SNRs. Table 3 gives the numerical results of RSHL, LFM and HFM.

We take Figure 10(a) as an example to analyze the performance of RSHL method under various SNRs. From Figure 10(a), it can be seen that, in the RSHL method, after matched filtering, the maximum points $t_{1}=20.8983$ and $t_{2}=19.8434$. Based 
Table 3 Results under Simulation Environment 2

\begin{tabular}{|c|c|c|c|c|c|c|c|c|c|c|}
\hline Speed $v$ & \multicolumn{2}{|c|}{ Peak moment } & Speed measurement & Ranging of & Speed measurement & Ranging error & Ranging $\mathrm{e}$ & single signal & Range accuracy & Provement ratio \\
\hline$(\mathrm{m} / \mathrm{s})$ & $t_{1}$ & $t_{2}$ & of RSHL $(\mathrm{m} / \mathrm{s})$ & $\mathrm{RSHL}(\mathrm{km})$ & error of RSHL & of RSHL & HFM & LFM & Compared to HFM & Compared to LFM \\
\hline 15 & 20.8983 & 19.8434 & 15.5126 & 14.9912 & $3.417333 \%$ & $0.058667 \%$ & $4.4915 \%$ & $0.783 \%$ & $98.69383 \%$ & $92.50745 \%$ \\
\hline$\frac{10}{10}$ & 20.5947 & 19.8966 & $\frac{1.5268}{10.2668}$ & 14.9943 & $2.668 \%$ & $0.038 \%$ & $2.9735 \%$ & $0.517 \%$ & $98.72204 \%$ & $92.6499 \%$ \\
\hline$\frac{5}{5}$ & 20.295 & 19.95 & 5.0735 & 14.998 & $1.47 \%$ & $0.013333 \%$ & $1.475 \%$ & $0.25 \%$ & $99.09605 \%$ & $94.66667 \%$ \\
\hline-15 & 19.1369 & 20.1323 & -14.6387 & 14.9967 & $2.408667 \%$ & $0.022 \%$ & $4.3155 \%$ & $0.6615 \%$ & $99.49021 \%$ & $96.67423 \%$ \\
\hline-10 & 19.4209 & 20.1027 & -10.0273 & $\frac{15.0068}{15.0068}$ & $0.273 \%$ & $0.045333 \%$ & $\frac{4.5955 \%}{2.895 \%}$ & $0.5135 \%$ & $98.43435 \%$ & $91.1717 \%$ \\
\hline-3 & 19.8249 & 20.0283 & -2.9916 & 15.0003 & $0.28 \%$ & $0.002 \%$ & $0.8755 \%$ & $0.1415 \%$ & $99.77156 \%$ & $98.58657 \%$ \\
\hline
\end{tabular}

Table 4 Results under Simulation Environment 3

\begin{tabular}{|c|c|c|c|c|c|c|c|c|c|c|}
\hline Speed $v$ & \multicolumn{2}{|c|}{ Peak moment } & Speed measurement & Ranging of & Speed measurement & Ranging error & Ranging & single signal & Range accuracy & rovement ratio \\
\hline$(\mathrm{m} / \mathrm{s})$ & $t_{1}$ & $t_{2}$ & of RSHL $(\mathrm{m} / \mathrm{s})$ & $\mathrm{RSHL}(\mathrm{km})$ & error of RSHL & of RSHL & HFM & LFM & Compared to HFM & Compared to LFM \\
\hline 15 & 20.4286 & 20.2249 & 16.0827 & 14.9837 & $7.218 \%$ & $0.108667 \%$ & $2.143 \%$ & $1.1245 \%$ & $94.92923 \%$ & $90.33645 \%$ \\
\hline 10 & 20.2839 & 20.1497 & 10.5902 & 14.9905 & $5.902 \%$ & $0.063333 \%$ & $1.4195 \%$ & $0.7485 \%$ & $95.53834 \%$ & $91.53863 \%$ \\
\hline 5 & 20.141 & 20.077 & 5.0526 & 14.9996 & $1.052 \%$ & $0.002667 \%$ & $0.705 \%$ & $0.385 \%$ & $99.62175 \%$ & $99.30736 \%$ \\
\hline-15 & 19.5881 & 19.7836 & -15.4286 & 15.0151 & $2.857333 \%$ & $0.100667 \%$ & $2.0595 \%$ & $1.082 \%$ & $95.11208 \%$ & $90.69624 \%$ \\
\hline-10 & 19.7236 & 19.8533 & -10.2406 & 15.0077 & $2.406 \%$ & $0.051333 \%$ & $1.382 \%$ & $0.7335 \%$ & $96.28558 \%$ & $93.00159 \%$ \\
\hline-3 & 19.9164 & 19.9514 & -2.7632 & 14.9953 & $7.893333 \%$ & $0.031333 \%$ & $0.418 \%$ & $0.243 \%$ & $92.50399 \%$ & $87.10562 \%$ \\
\hline
\end{tabular}

on Equation (21), $R$ is equal to $14.9912 \mathrm{~km}$. According to Equation (20), the value of $v$ is $15.5126 \mathrm{~m} / \mathrm{s}$. The speed measurement error and the ranging error of RSHL are $3.417333 \%$ and $0.058667 \%$, respectively. The ranging error of HFM and LFM are $4.4915 \%$ and $0.783 \%$, respectively. It can be seen from Table 3 that, compared with HFM and LFM, the range measurement accuracy of RSHL is improved by $98.69383 \%$ and $92.50745 \%$, respectively.

Figure 11 shows the performance analysis under the simulation environment 1 under various SNRs. Table 4 gives the numerical results of RSHL, LFM and HFM.

We take Figure 11(a) as an example to analyze the performance of RSHL method under various SNRs. From Figure 11(a), it can be seen that, in the RSHL method, after matched filtering, the maximum points $t_{1}=20.4286$ and $t_{2}=20.2249$. Based on Equation (21), $R$ is equal to $14.9837 \mathrm{~km}$. According to Equation (20), the value of $v$ is $16.0827 \mathrm{~m} / \mathrm{s}$. The speed measurement error and the ranging error of RSHL are $7.218 \%$ and $0.108667 \%$, respectively. The ranging error of HFM and LFM are $2.143 \%$ and $1.1245 \%$, respectively. It can be seen from Table 4 that, compared with HFM and LFM, the range measurement accuracy of RSHL is improved by $94.92923 \%$ and $90.33645 \%$, respectively.

Figure 12 shows the performance analysis under the simulation environment 4 under various $v$, and Table 5 gives the numerical results of RSHL, LFM and HFM.

We take Figure 12(a) as an example to analyze the performance of RSHL method under various $v$. From Figure 12(a), it can be seen that, in the RSHL method, after matched filtering, the maximum points $t_{1}=20.44$ and $t_{2}=19.57$. Based on Equation (21), $R$ is equal to $15.0041 \mathrm{~km}$. According to Equation (20), the value of $v$ is $14.9655 \mathrm{~m} / \mathrm{s}$. The ranging error and the speed measurement error of RSHL are $0.027 \%$ and $0.23 \%$, respectively. The ranging error of LFM and HFM are $2.118 \%$ and $2.222 \%$, respectively. It can be seen from Table 5 that, compared with LFM and HFM, the range measurement accuracy of RSHL is improved by $98.70947 \%$ and $98.76988 \%$, respectively.

Table 5 Results under Simulation Environment 4

\begin{tabular}{|c|c|c|c|c|c|c|c|c|c|c|}
\hline Speed $v$ & \multicolumn{2}{|c|}{ Peak moment } & Speed measurement & Ranging of & Speed measurement & Ranging error & \multicolumn{2}{|c|}{ Ranging error of single signal } & \multicolumn{2}{|c|}{ Range accuracy improvement ratio } \\
\hline$\frac{\mathrm{s} / \mathrm{s})}{(\mathrm{s})}$ & $t_{1}$ & $t_{2}$ & of RSHL $(\mathrm{km})$ & $\operatorname{RSHL}(\mathrm{km})$ & error of RSHL & of RSHL & LFM & HFM & Compared to LFM & Compared to HFM \\
\hline 15 & 20.4444 & 19.5764 & 15.0041 & 14.9655 & $0.23 \%$ & $0.027333 \%$ & $2.222 \%$ & $2.118 \%$ & $98.76988 \%$ & $98.70947 \%$ \\
\hline 10 & 20.296 & 19.7187 & 15.003 & 9.9532 & $0.468 \%$ & $0.02 \%$ & $1.48 \%$ & $1.4065 \%$ & $98.64865 \%$ & $98.57803 \%$ \\
\hline 5 & 20.1479 & 19.8603 & 15.0018 & 4.9581 & $0.838 \%$ & $0.012 \%$ & $0.7395 \%$ & $0.6985 \%$ & $98.37728 \%$ & $98.28203 \%$ \\
\hline-15 & 19.5641 & 20.4147 & 14.9957 & -14.665 & $2.233333 \%$ & $0.028667 \%$ & $2.1795 \%$ & $2.0735 \%$ & $98.68471 \%$ & $98.61747 \%$ \\
\hline-10 & 19.71 & 20.2787 & 14.9982 & -9.8054 & $1.946 \%$ & $0.012 \%$ & $1.45 \%$ & $1.3935 \%$ & $99.17241 \%$ & $99.13886 \%$ \\
\hline-3 & 19.9119 & 20.0834 & 14.999 & -2.9581 & $1.396667 \%$ & $0.006667 \%$ & $0.4405 \%$ & $0.417 \%$ & $98.48657 \%$ & $98.40128 \%$ \\
\hline
\end{tabular}




\section{Conclusion}

In this paper, a Ranging and Speed measurement method by jointing Hyperbolic frequency modulation and Linear frequency modulation (RSHL) is proposed. On one hand, the RSHL method can not only accurately measure the speed, but also greatly decrease the ranging error caused by Doppler frequency shift in unidirectional modulation HFM. On the other hand, RSHL method relaxes the bandwidth and frequency requirements, optimize the transmission signal form, reduce the operation cost, improve the measurement accuracy, and make full use of frequency band resources or pulse width resources.

To improve the accuracy of combined signals for speed measurement and ranging, that is, at the same speed, the matched filtering delay $t_{0}$ caused by the Doppler of the target is as large as possible. According to Equation (9), there are three ways to improve $t_{0}: 1$ ) Increase the frequency $\left.f_{1} ; 2\right)$ Decrease the bandwidth $\left(f_{1}-f_{0}\right) ; 3$ ) Increase the pulse width $T$. Each way requires additional hardware costs, and the complexity of the entire system must be considered comprehensively.

\section{Abbreviations}

LFM: Linear Frequency Modulation; HFM: Hyperbolic Frequency Modulation; FM: Frequency Modulation; RSHL: Ranging and Speed measurement method by jointing Hyperbolic frequency modulation and Linear frequency modulation; SNR: Signal-to-Noise Ratio.

\section{Acknowledgements}

We acknowledge the support of Yuankun Peng from Hangzhou Institute of Applied Acoustics for his critical remarks.

\section{Authors' contributions}

CS conceived the study, conducted the experiments, supervised the study and wrote the paper. PL reviewed and edited the manuscript. The authors read and approved the final manuscript.

\section{Funding}

This work was supported in part by the Shandong Smart Ocean Ranch Engineering Technology Collaborative Innovation Center, in part by the research fund for high-level talents of Qingdao Agricultural University (NO.1119048), in part by the Shandong Agricultural Science and Technology Service Project (NO.2019FW037-4), in part by Shandong Technology Innovation Guidance Program (NO.2020LYXZ023), in part by Horizontal Project (NO.20193702010792), in part by Experimental technical project of Qingdao Agricultural University (NO.SYJK18-01), and in part by Ministry of Education Industry-University Cooperation Collaborative Education Project (NO.201902005027 and NO.201901029013).

\section{Availability of data and materials}

Please contact the corresponding author for simulation results.

\section{Declarations}

\section{Consent for publication}

Informed consent was obtained from all authors included in the study.

\section{Competing interests}

The authors declare that they have no competing interests.

\footnotetext{
Author details

1, Hangzhou Institute of Applied Acoustics, Hangzhou, China. ${ }^{2}$ College of Science and Information, Qingdao Agricultural University, Qingdao, China.
} 


\section{References}

1. De Witte, E., Griffiths, H.: Improved ultra-low range sidelobe pulse compression waveform design. Electronics Letters 40(22), 1448-1450 (2004)

2. Rao, M.N., Rajeswari, K.R.: Target detection with cross ambiguity function using binary sequences with high discrimination. International Journal of Computer Applications 16(4), 8-12 (2011)

3. Govoni, M.-A.: Linear Frequency Modulation of Stochastic Radar Waveform. Stevens Institute of Technology, ??? (2011)

4. Huo, K., Deng, B., Liu, Y., Jiang, W., Mao, J.: High resolution range profile analysis based on multicarrier phase-coded waveforms of ofdm radar. Journal of Systems Engineering and Electronics 22(3), 421-427 (2011)

5. Skolnik, M.I.: Introduction to Radar Systems. McGRAW-HILL book company, ??? (1990)

6. Patton, L., Frost, S., Rigling, B.: Efficient design of radar waveforms for optimised detection in coloured noise IET Radar, Sonar \& Navigation 6(1), 21-29 (2012)

7. Li, Q.: Digital Sonar Design in Underwater Acoustics: Principles and Applications. Anhui Education Press, ??? (2003)

8. Łuszczyk, M., Łabudzinski, A.: Sidelobe level reduction for complex radar signals with small base. In: 2012 13th International Radar Symposium, pp. 146-149 (2012). IEEE

9. Ai, X., Li, Y., Wang, X., Xiao, S.: Some results on characteristics of bistatic high-range resolution profiles for target classification. IET Radar, Sonar \& Navigation 6(5), 379-388 (2012)

10. White, P.R., Locke, J.: Performance of methods based on the fractional fourier transform for the detection of linear frequency modulated signals. IET signal processing 6(5), 478-483 (2012)

11. Klinger, O., Stern, Y., Schneider, T., Jamshidi, K., Zadok, A.: Long microwave-photonic variable delay of linear frequency modulated waveforms. IEEE Photonics Technology Letters 24(3), 200-202 (2011)

12. Yuhong, P., Qi, Y., Shichuang, W.: Research on hyperbolic frequency modulation signal speed and ranging method. Acoustics and Electrical Engineering (04), 21-24 (2014)

13. Shi, C., Ding, L., Wang, F., Salous, S., Zhou, J.: Joint target assignment and resource optimization framework for multitarget tracking in phased array radar network. IEEE Systems Journal PP(99), 1-12 (2020)

14. Liu, Y., Liao, G., Chen, Y., Xu, J., Yin, Y.: Super-resolution range and velocity estimations with ofdm integrated radar and communications waveform. IEEE Transactions on Vehicular Technology PP(99), 1-1 (2020)

15. Liu, Y., Liao, G., Yang, Z., Xu, J.: Joint range and angle estimation for an integrated system combining mimo radar with ofdm communication. Multidimensional Systems and Signal Processing (3), 1-27 (2018)

16. Chiriyath, A.R., Paul, B., Bliss, D.W.: Simultaneous radar detection and communications performance with clutter mitigation. In: 2017 IEEE Radar Conference (RadarConf17) (2017)

17. Whyland, W.P.: Doppler consistent hyperbolic frequency modulation. Google Patents. US Patent 5,077,702 (1991)

18. Doisy, Y., Deruaz, L., Beerens, S.P., Been, R.: Target doppler estimation using wideband frequency modulated signals. IEEE transactions on signal processing 48(5), 1213-1224 (2000)

19. Lee, C.-E., Lee, H.-W., Kim, K.-M., Kim, W.-S., Chun, S.-Y., Lee, S.-K.: Underwater communication with amplitude-hyperbolic frequency modulation. In: 2014 Sixth International Conference on Ubiquitous and Future Networks (ICUFN), pp. 560-561 (2014). IEEE

20. Wang, F., Du, S., Sun, W., Huang, Q., Su, J.: A method of velocity estimation using composite hyperbolic frequency-modulated signals in active sonar. The Journal of the Acoustical Society of America 141(5), 3117-3122 (2017)

21. Murray, J.J.: On the doppler bias of hyperbolic frequency modulation matched filter time of arrival estimates. IEEE Journal of Oceanic Engineering 44(2), 446-450 (2019). doi:10.1109/JOE.2018.2819779

22. Huang, S., Fang, S., Han, N.: An improved velocity estimation method for wideband multi-highlight target echoes in active sonar systems. Sensors 18(9), 2794 (2018)

23. Kim, M., Im, T., Cho, Y., Kim, K., Ko, H.: Hfm design for timing synchronization in underwater communications systems. In: OCEANS 2017 - Aberdeen, pp. 1-4 (2017). doi:10.1109/OCEANSE.2017.8084893

24. Jedel, A., Marszal, J., Salamon, R.: Continuous wave sonar with hyperbolic frequency modulation keyed by pseudo-random sequence. Hydroacoustics 19 (2016)

25. Diamant, R., Feuer, A., Lampe, L.: Choosing the right signal: Doppler shift estimation for underwater acoustic signals. In: Proceedings of the Seventh ACM International Conference on Underwater Networks and Systems. WUWNet '12, pp. 27-1278. ACM, New York, NY, USA (2012). doi:10.1145/2398936.2398971. http://doi.acm.org/10.1145/2398936.2398971

26. Tan, T.: Sonar Technology. Harbin Engineering University Press, ??? (2010)

27. Guo, R., Cai, Z.M., Yao, Z.X.: Sidelobe suppression method of linear frequency modulated signal without mainlobe widening. Journal of Electronics \& Information Technology 36(2), 298-303 (2014) 


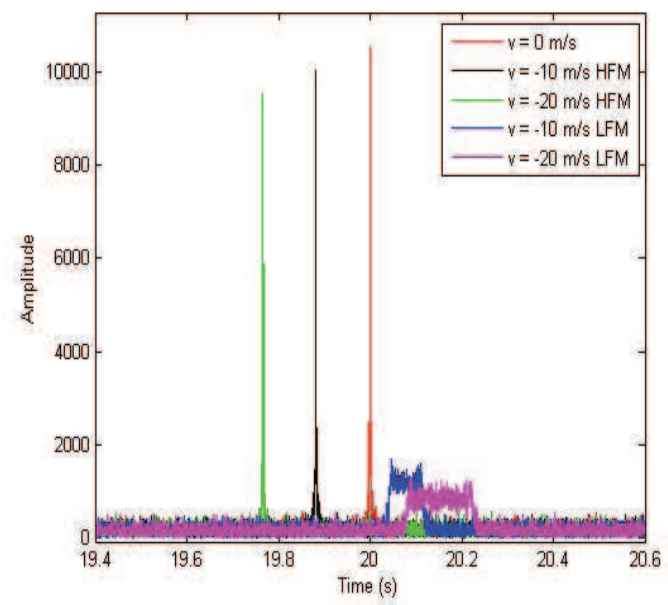

(a)

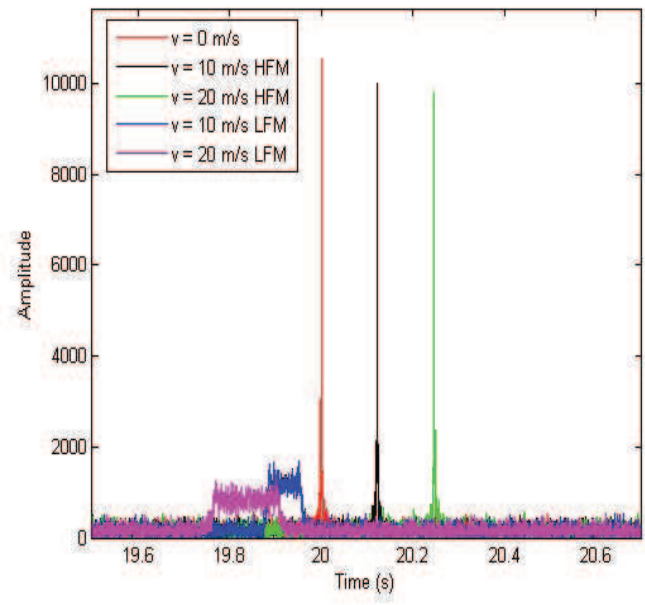

(b)

Figure 7 Time delay of the target motion related to the FM signal with the positive and negative slope.

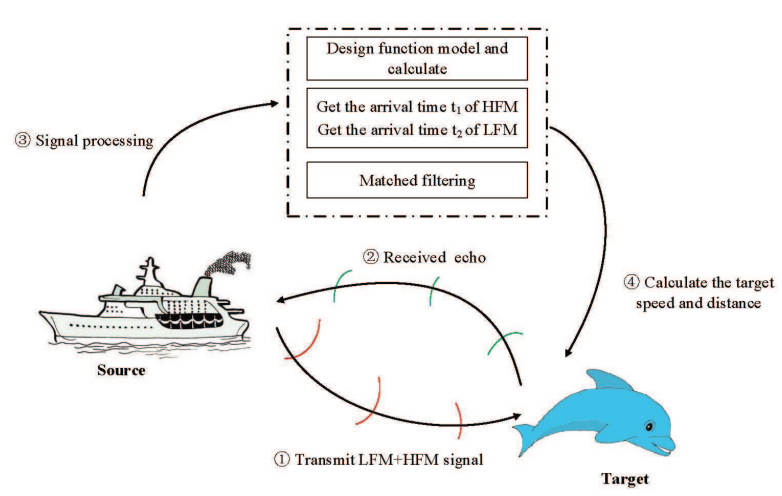

Figure 8 The works processing of the RSHL method. 


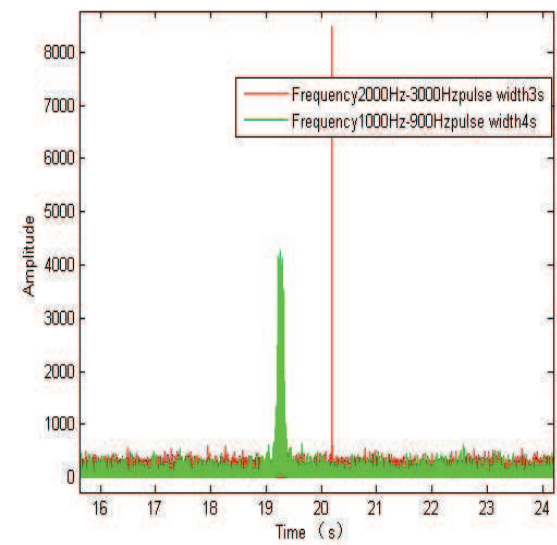

(a) $v=15 \mathrm{~m} / \mathrm{s}$.

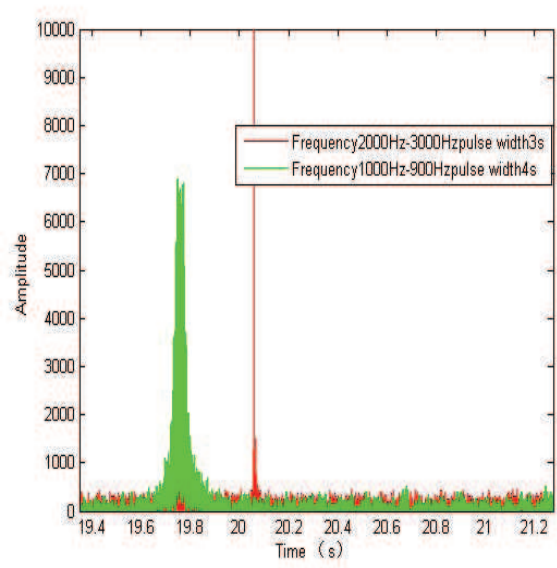

(c) $v=5 \mathrm{~m} / \mathrm{s}$.

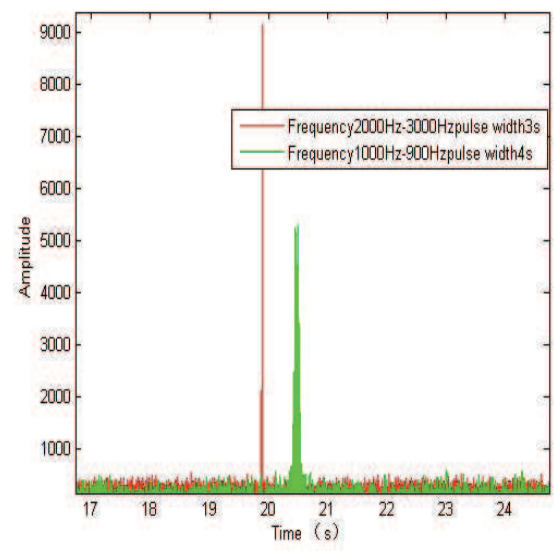

(e) $v=-10 \mathrm{~m} / \mathrm{s}$.

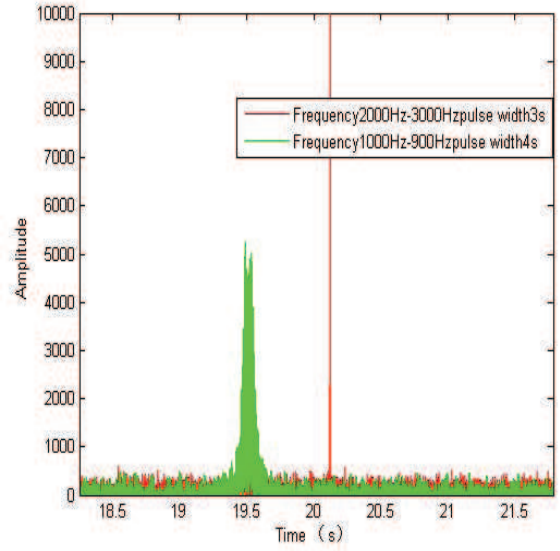

(b) $v=10 \mathrm{~m} / \mathrm{s}$.

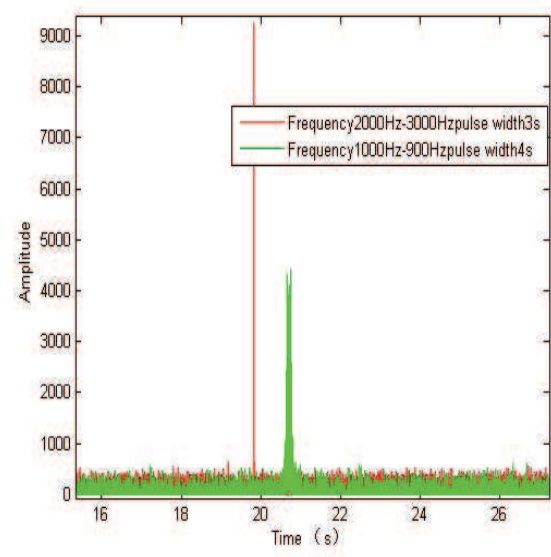

(d) $v=-15 \mathrm{~m} / \mathrm{s}$.

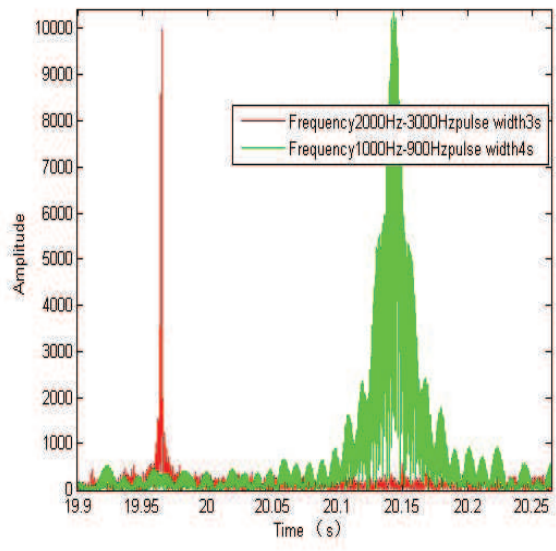

(f) $v=-3 m / s$.

Figure 9 The output of matched filtering at various $v$ under Simulation Environment 1 . 


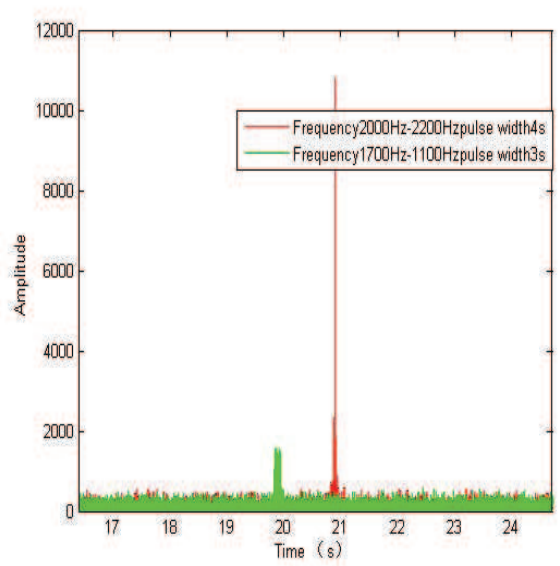

(a) $v=15 \mathrm{~m} / \mathrm{s}$.

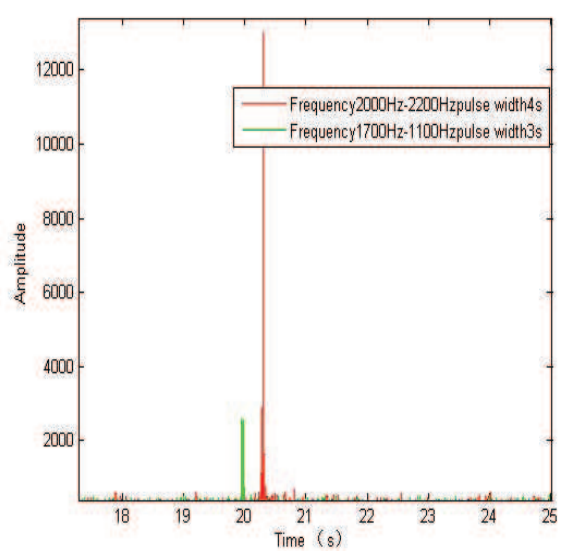

(c) $v=5 \mathrm{~m} / \mathrm{s}$.

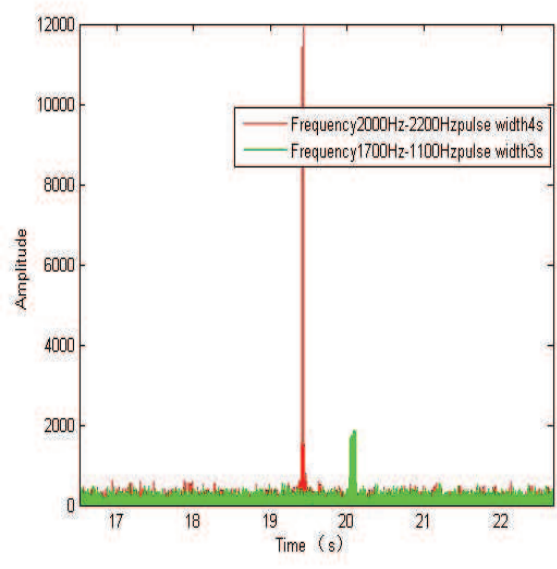

(e) $v=-10 \mathrm{~m} / \mathrm{s}$.

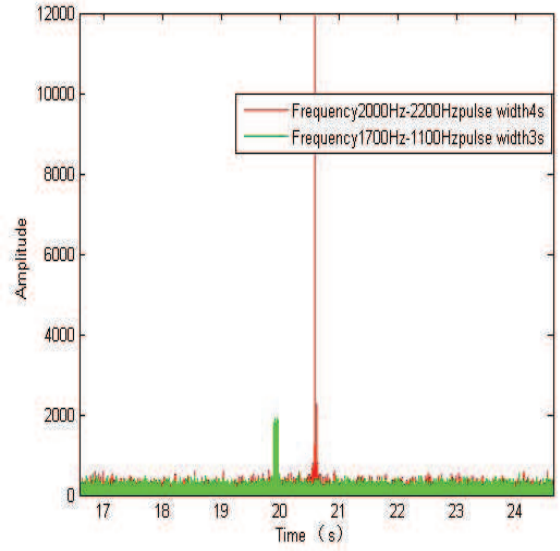

(b) $v=10 \mathrm{~m} / \mathrm{s}$.

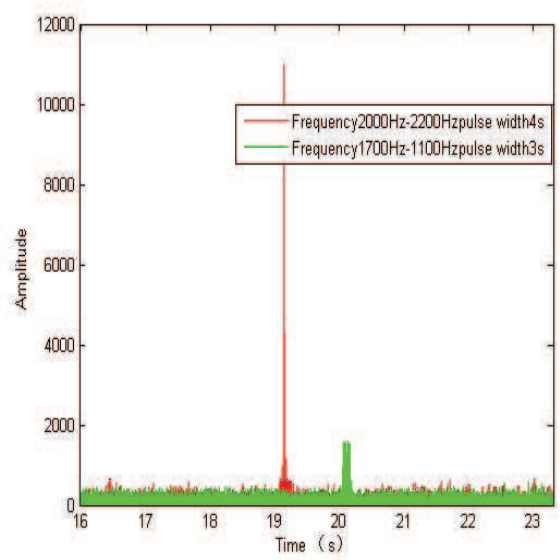

(d) $v=-15 \mathrm{~m} / \mathrm{s}$.

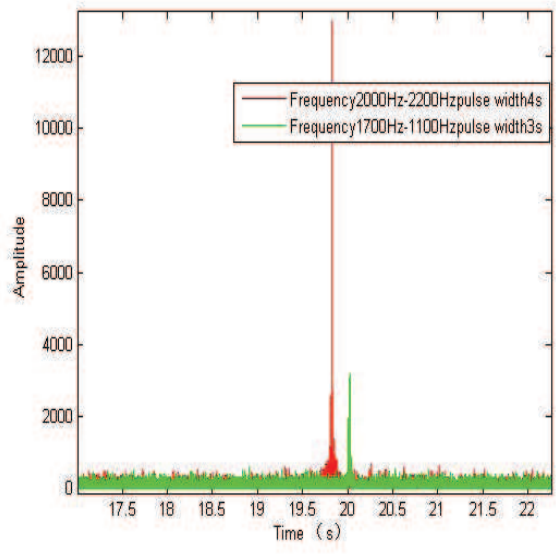

(f) $v=-3 m / s$.

Figure 10 The output of matched filtering at various $v$ under Simulation Environment 2. 


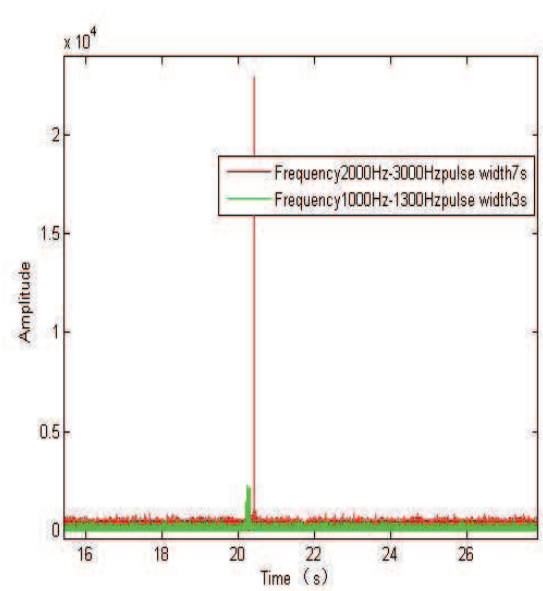

(a) $v=15 \mathrm{~m} / \mathrm{s}$.

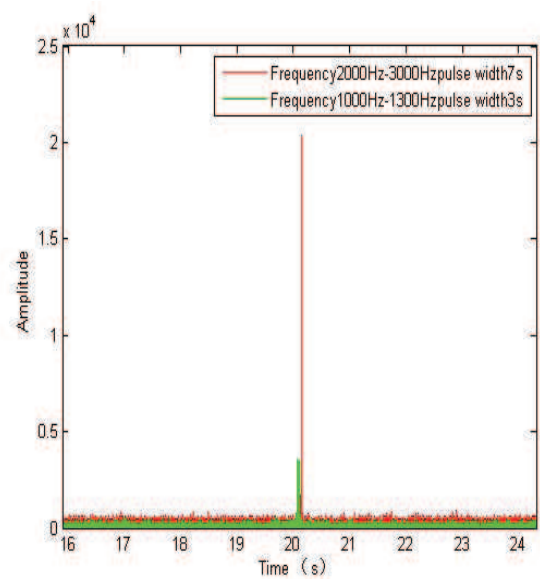

(c) $v=5 \mathrm{~m} / \mathrm{s}$.

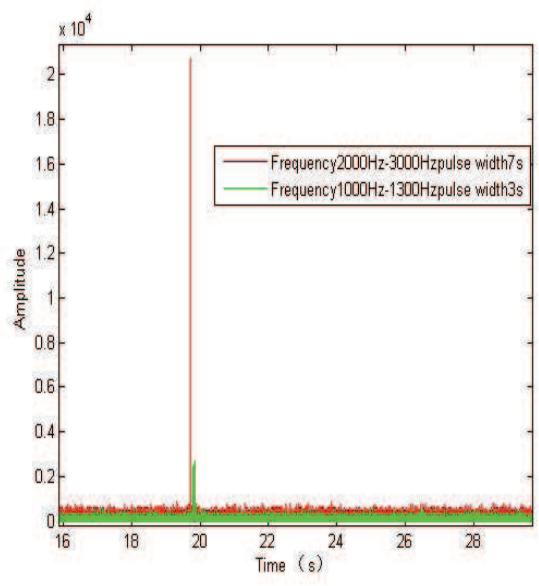

(e) $v=-10 \mathrm{~m} / \mathrm{s}$.

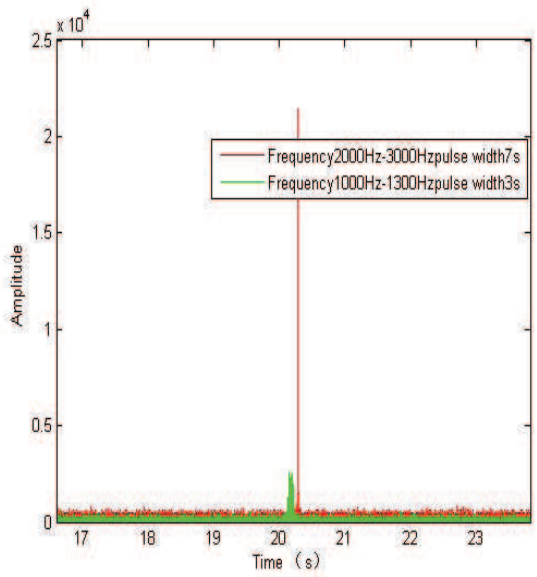

(b) $v=10 \mathrm{~m} / \mathrm{s}$.

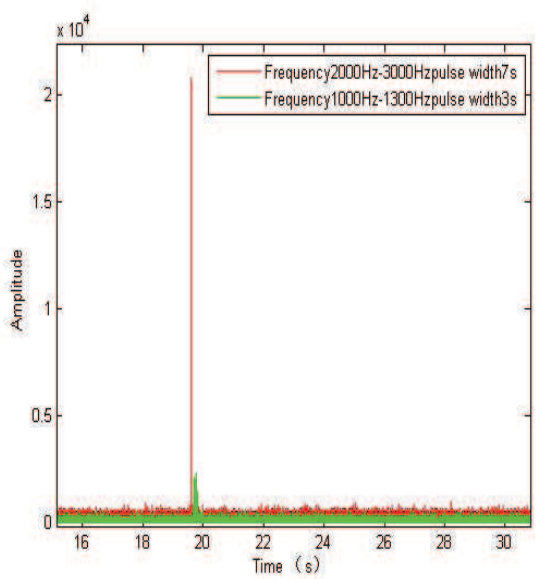

(d) $v=-15 \mathrm{~m} / \mathrm{s}$.

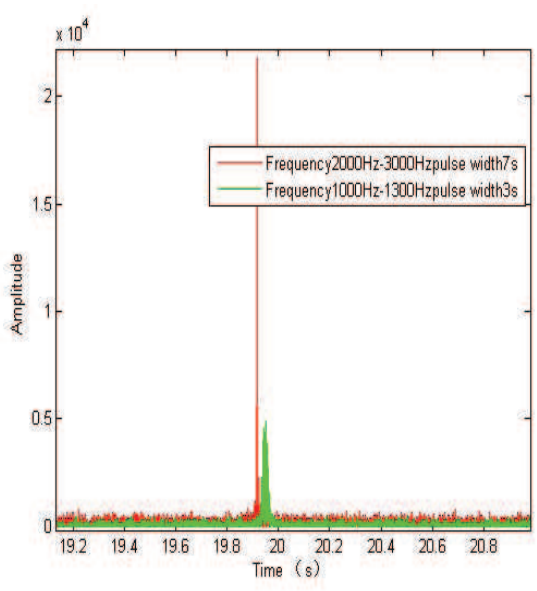

(f) $v=-3 m / s$.

Figure 11 The output of matched filtering at various $v$ under Simulation Environment 3. 


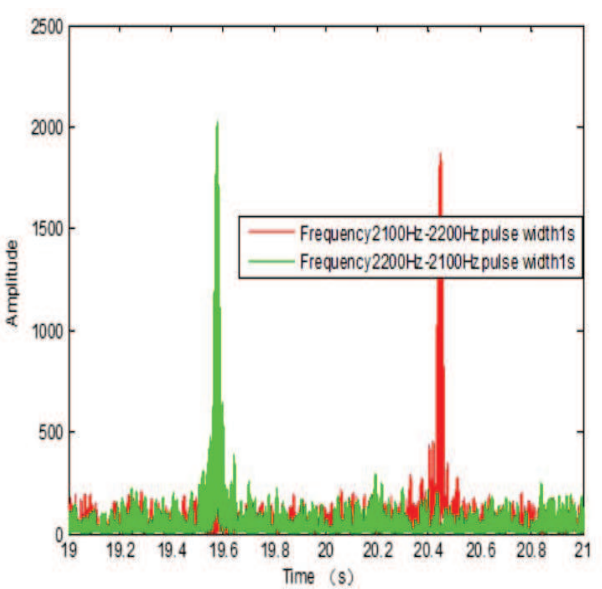

(a) $v=15 \mathrm{~m} / \mathrm{s}$.

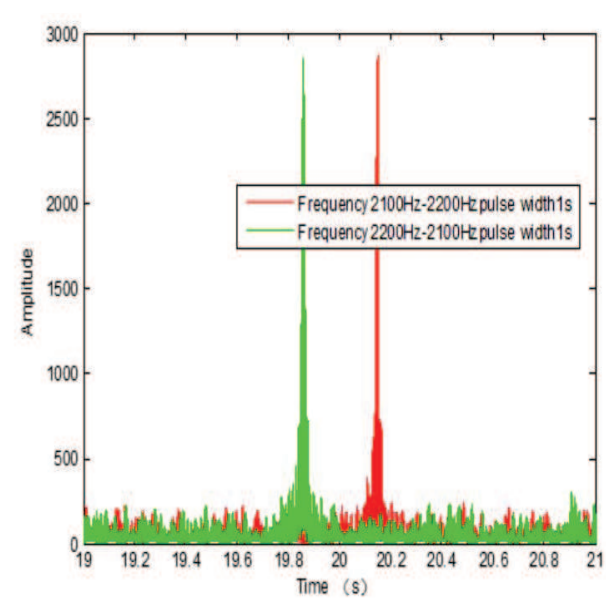

(c) $v=5 \mathrm{~m} / \mathrm{s}$.

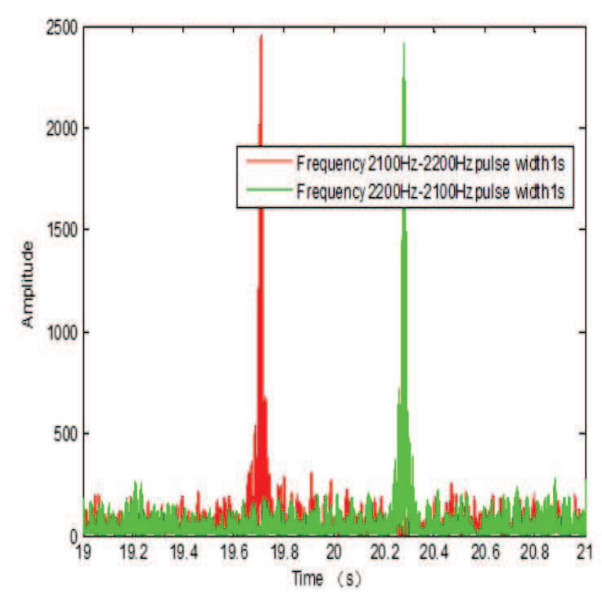

(e) $v=-10 \mathrm{~m} / \mathrm{s}$.

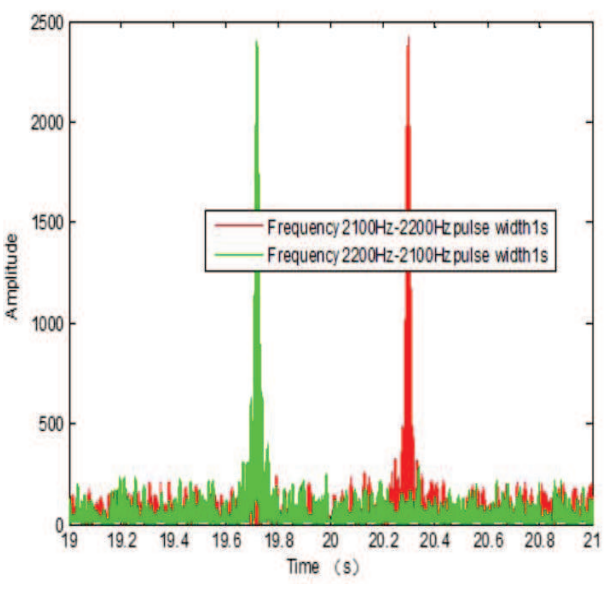

(b) $v=10 \mathrm{~m} / \mathrm{s}$.

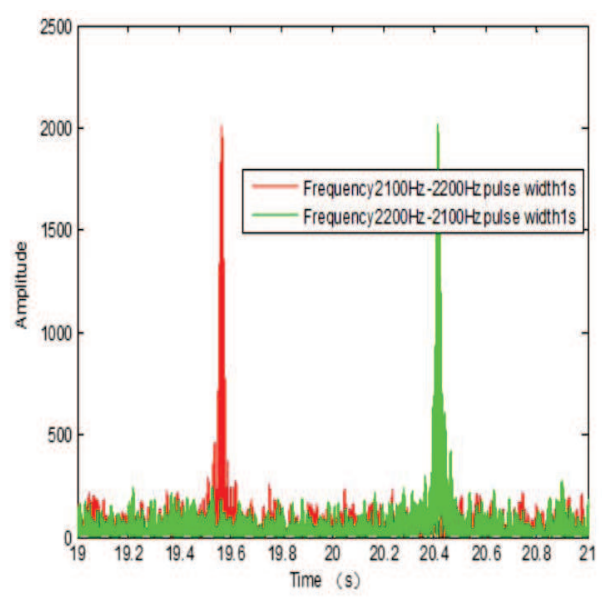

(d) $v=-15 \mathrm{~m} / \mathrm{s}$.

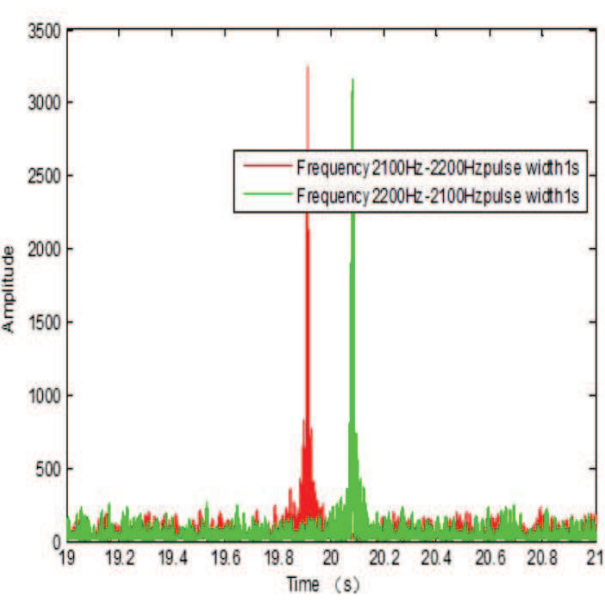

(f) $v=-3 m / s$.

Figure 12 The output of matched filtering at various $v$ under Simulation Environment 4. 
Figures

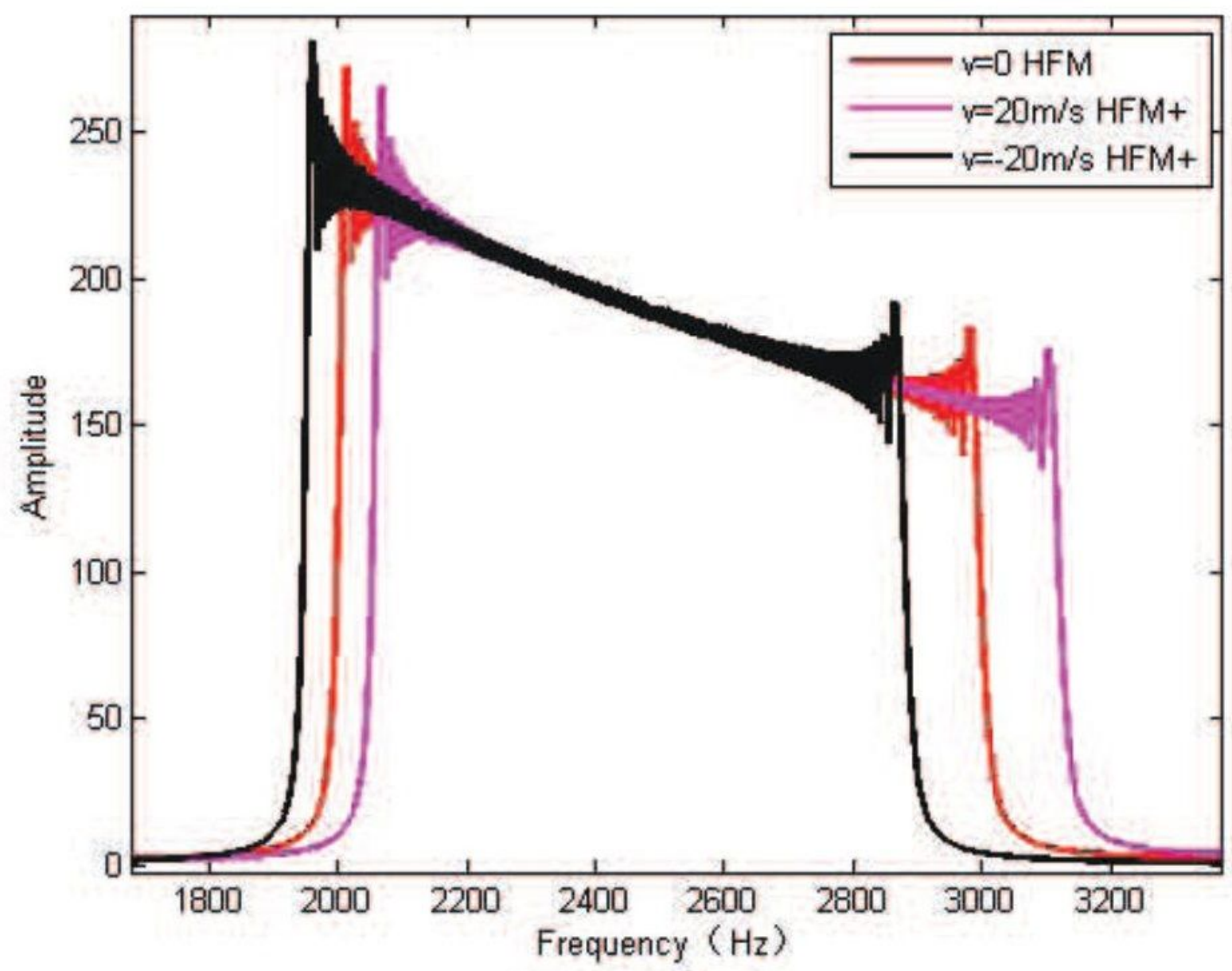

Figure 1

Please see the Manuscript PDF file for the complete figure caption 


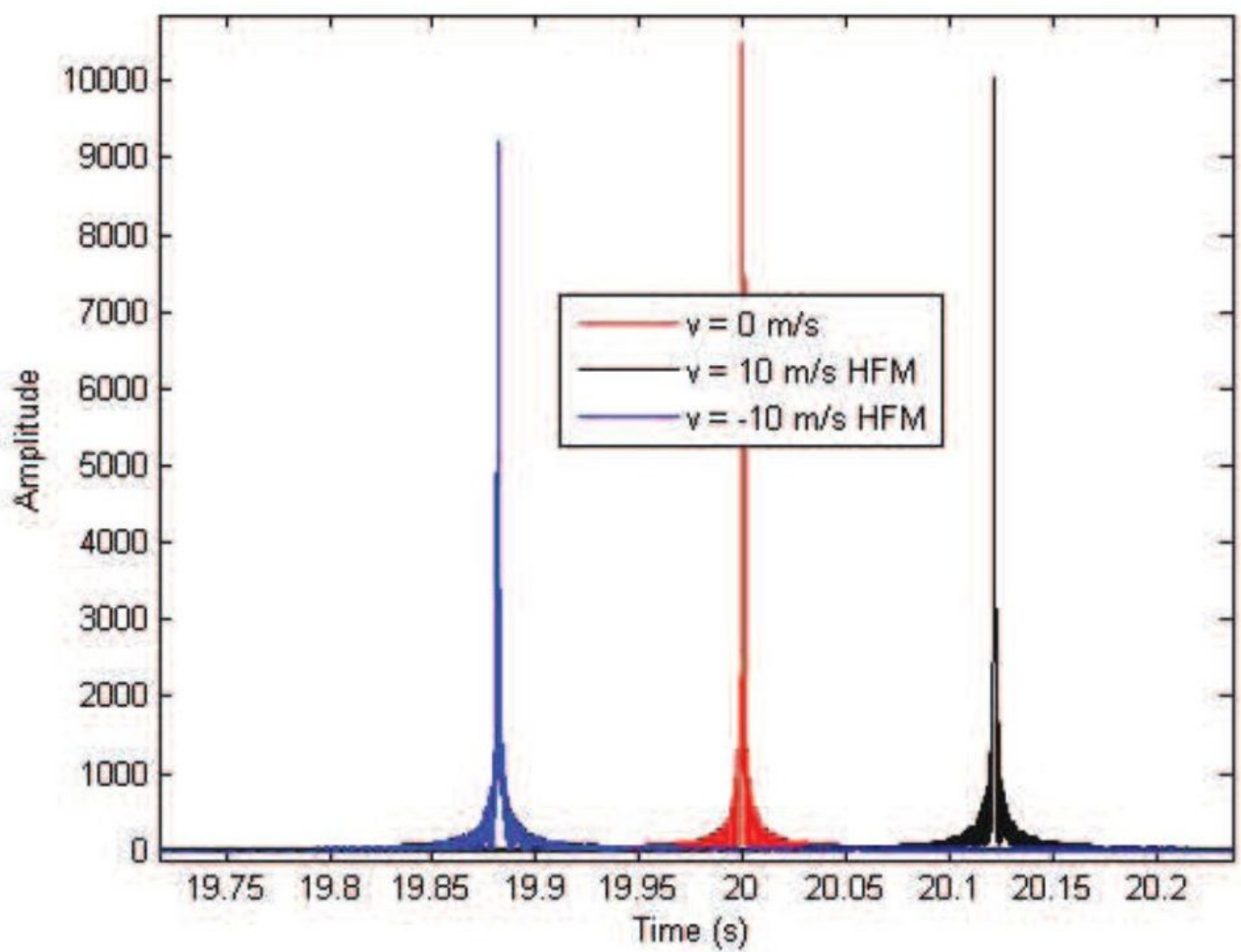

Figure 2

Please see the Manuscript PDF file for the complete figure caption 


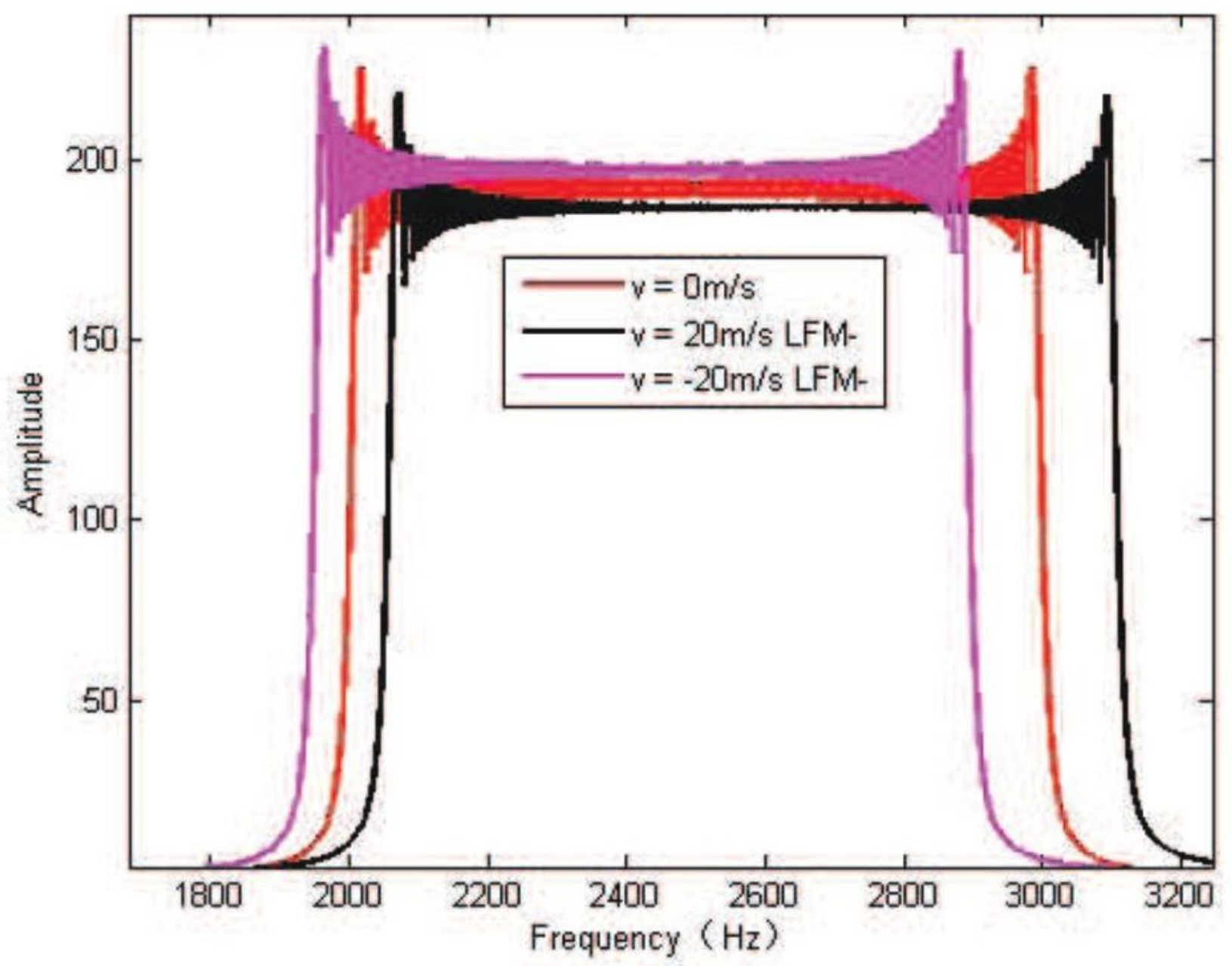

Figure 3

Please see the Manuscript PDF file for the complete figure caption 


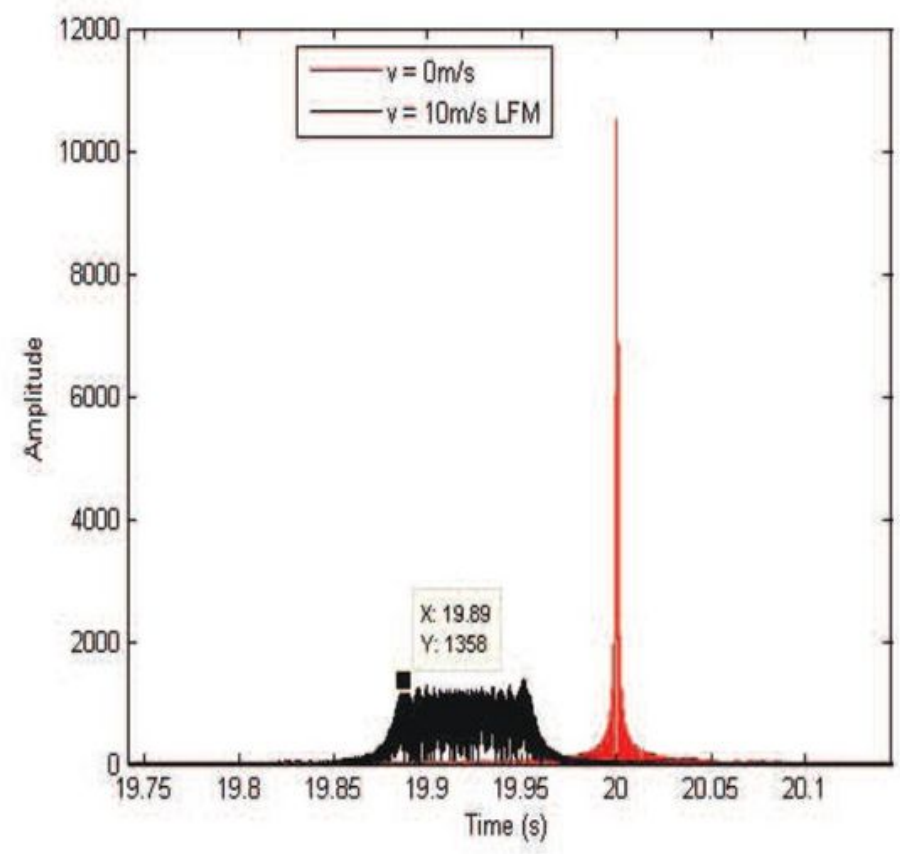

(a)

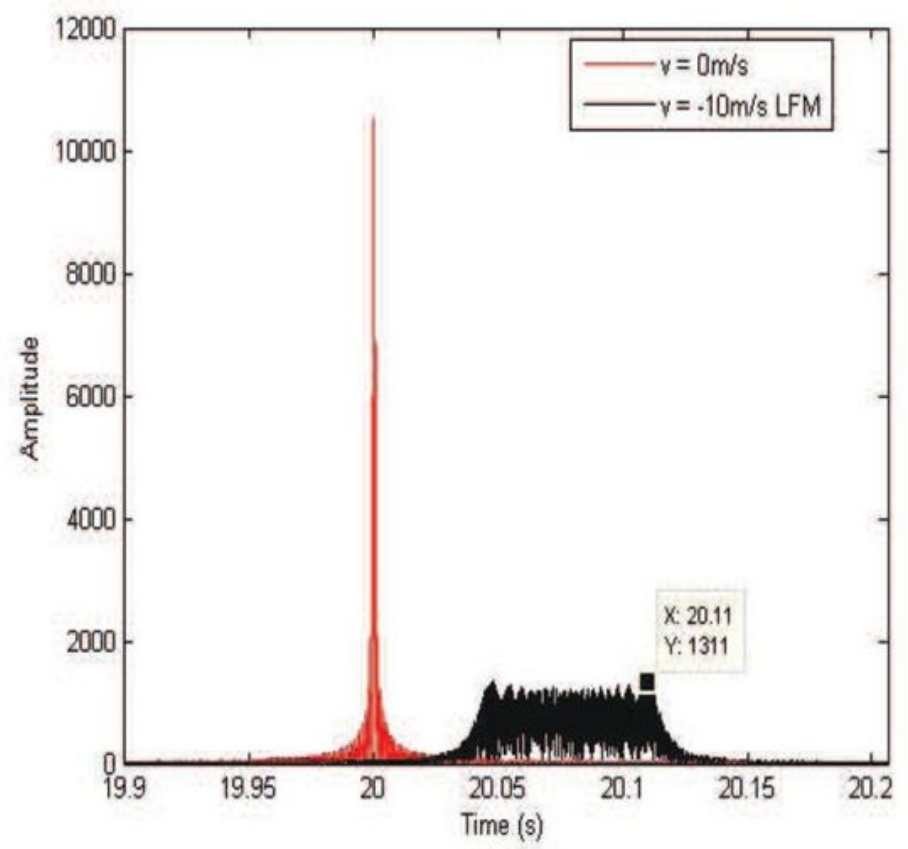

(b)

\section{Figure 4}

Please see the Manuscript PDF file for the complete figure caption 


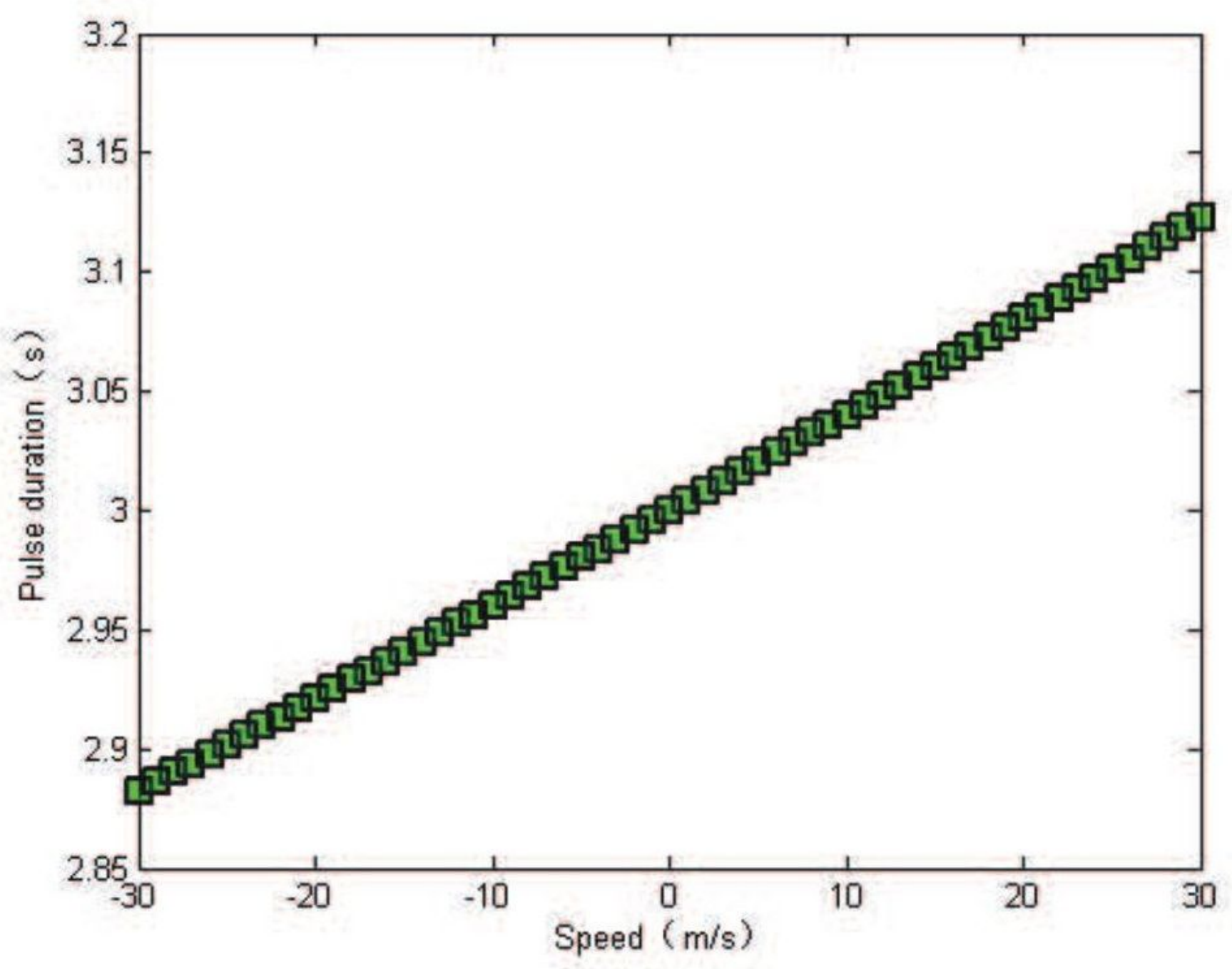

Figure 5

Please see the Manuscript PDF file for the complete figure caption 


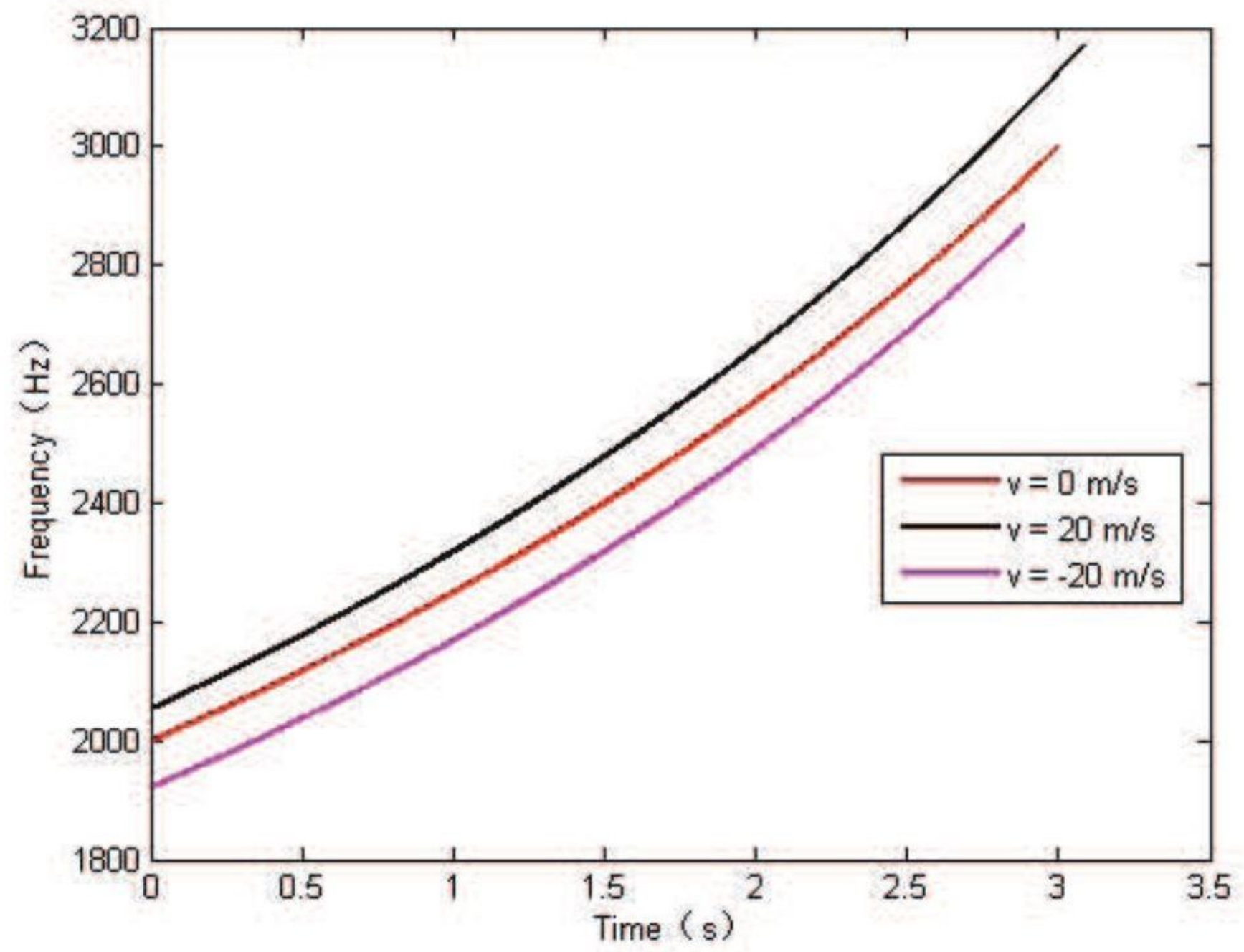

Figure 6

Please see the Manuscript PDF file for the complete figure caption 


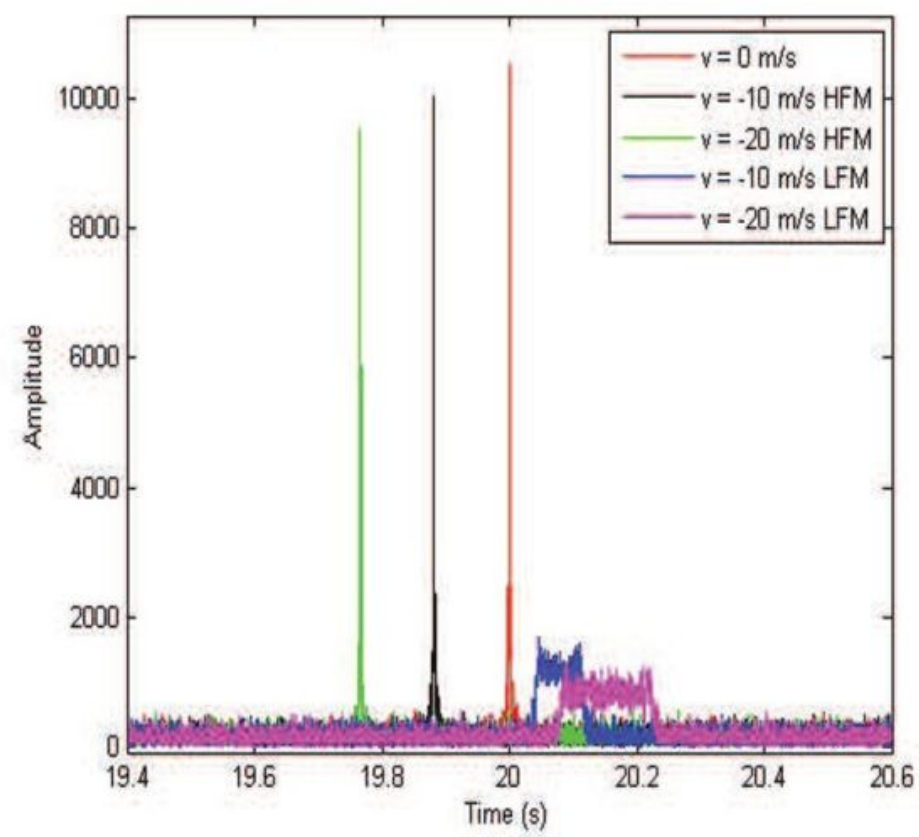

(a)

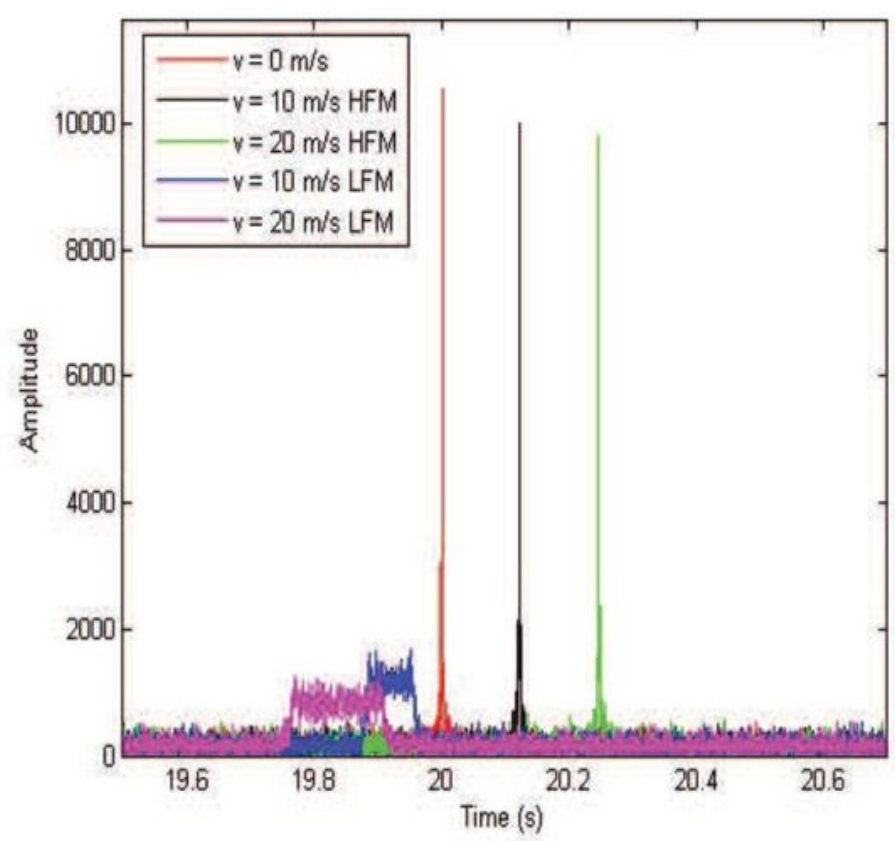

(b)

Figure 7

Please see the Manuscript PDF file for the complete figure caption 


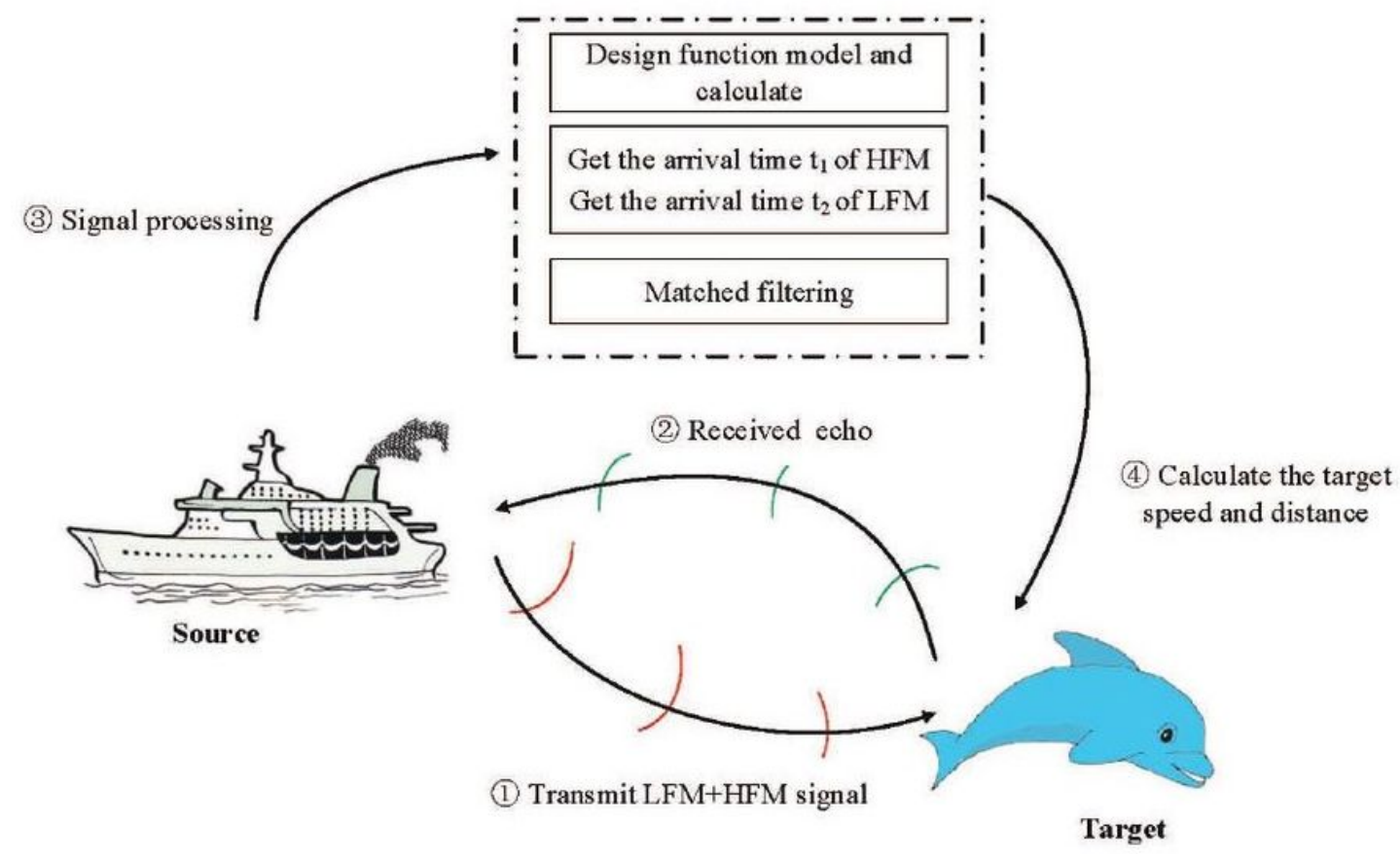

Figure 8

Please see the Manuscript PDF file for the complete figure caption 


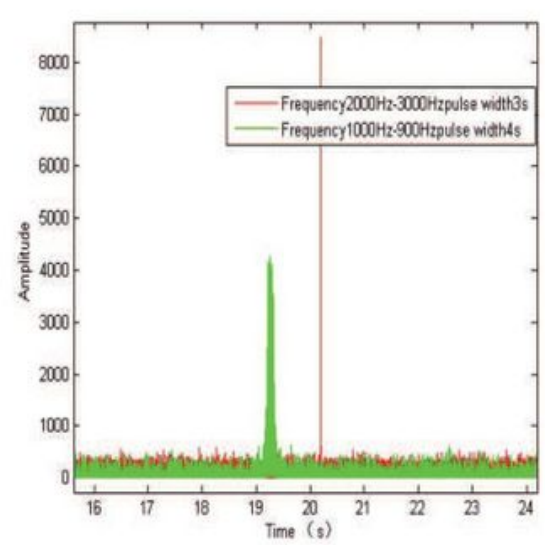

(a) $v=15 \mathrm{~m} / \mathrm{s}$.

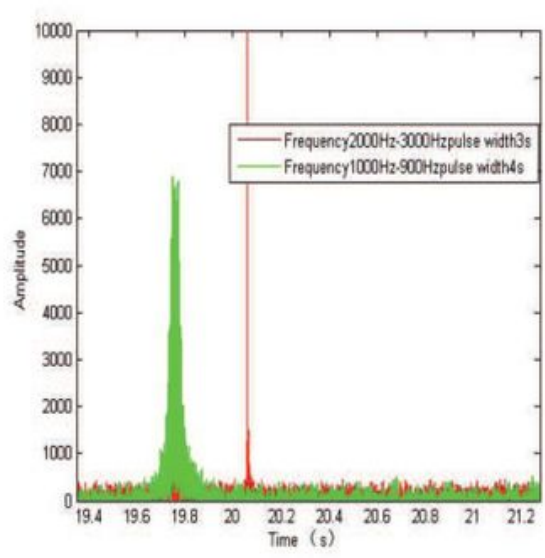

(c) $v=5 \mathrm{~m} / \mathrm{s}$.

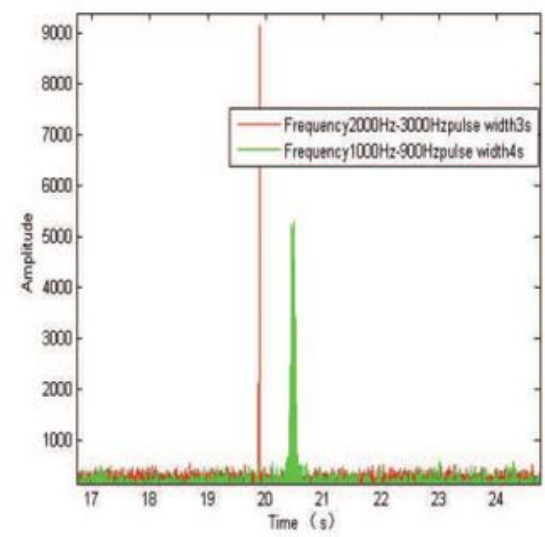

(e) $v=-10 \mathrm{~m} / \mathrm{s}$.

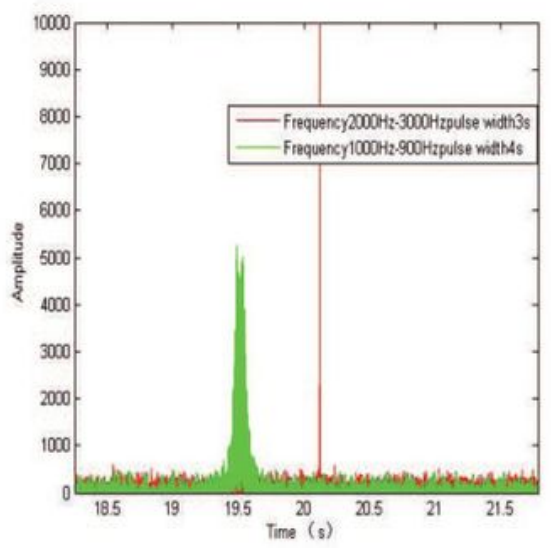

(b) $v=10 \mathrm{~m} / \mathrm{s}$.

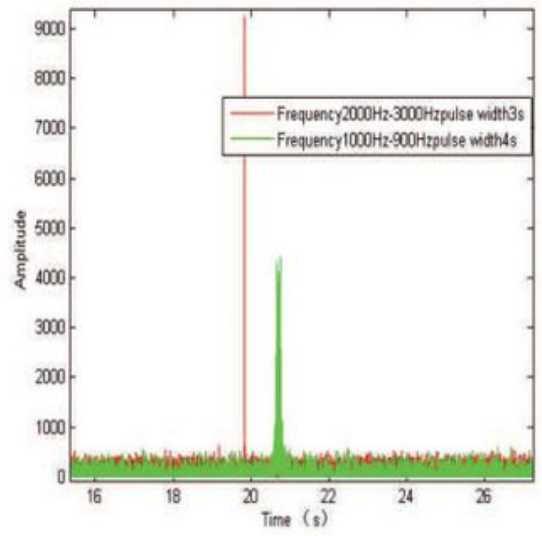

(d) $v=-15 \mathrm{~m} / \mathrm{s}$.

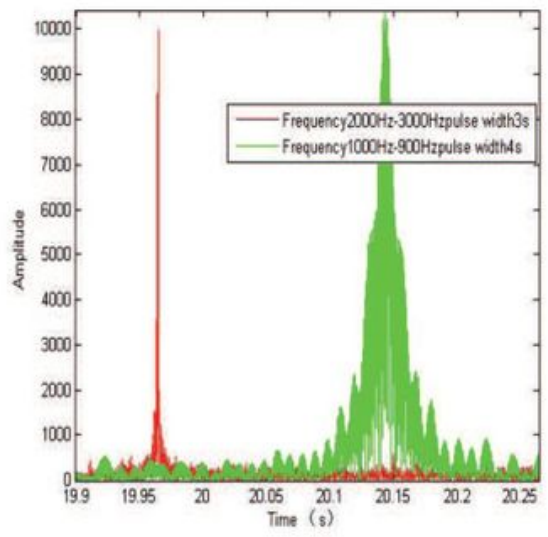

(f) $v=-3 \mathrm{~m} / \mathrm{s}$.

Figure 9

Please see the Manuscript PDF file for the complete figure caption 


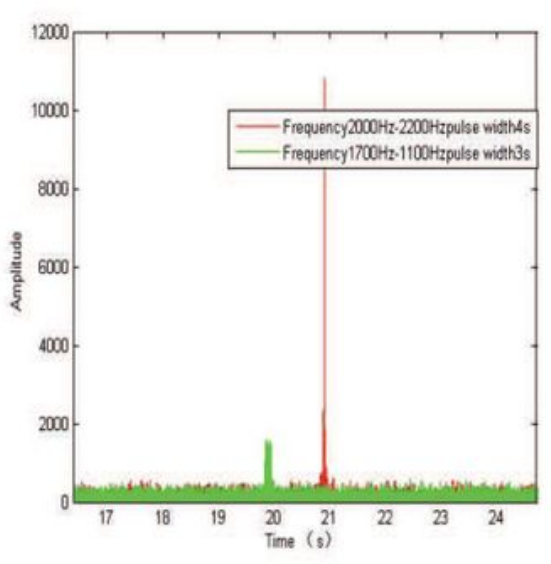

(a) $v=15 \mathrm{~m} / \mathrm{s}$.

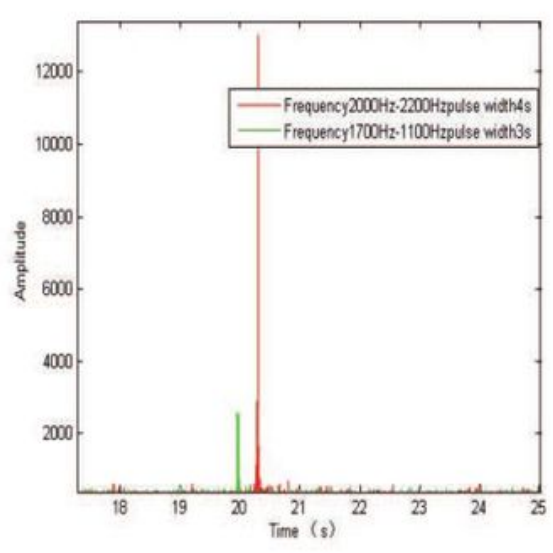

(c) $v=5 \mathrm{~m} / \mathrm{s}$.

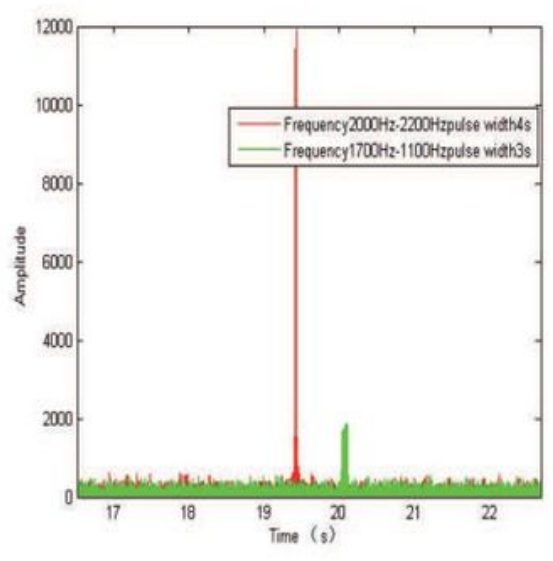

(e) $v=-10 \mathrm{~m} / \mathrm{s}$.

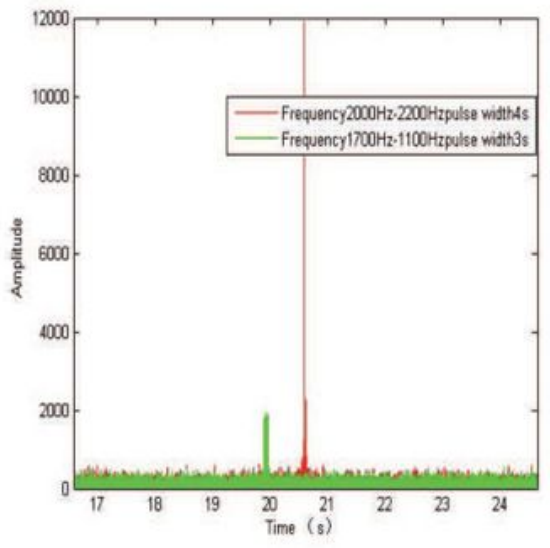

(b) $v=10 \mathrm{~m} / \mathrm{s}$.

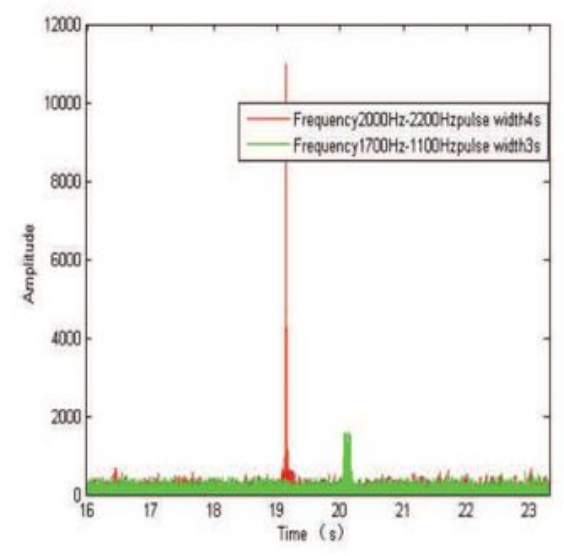

(d) $v=-15 \mathrm{~m} / \mathrm{s}$.

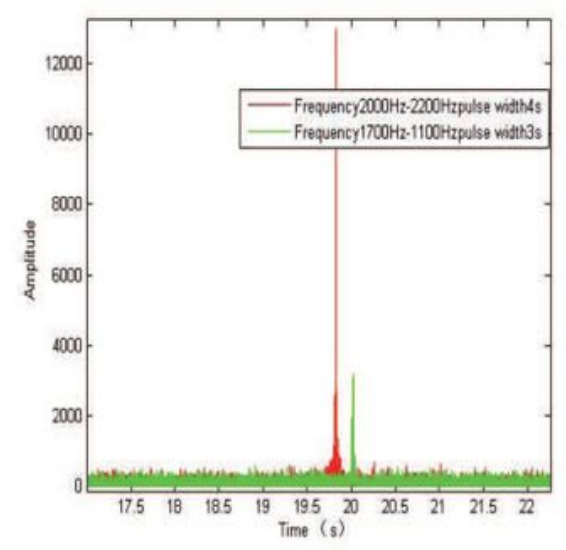

(f) $v=-3 m / s$.

\section{Figure 10}

Please see the Manuscript PDF file for the complete figure caption 


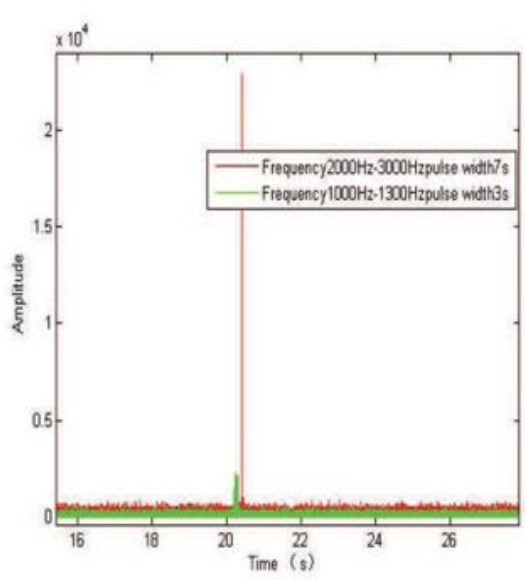

(a) $v=15 \mathrm{~m} / \mathrm{s}$.

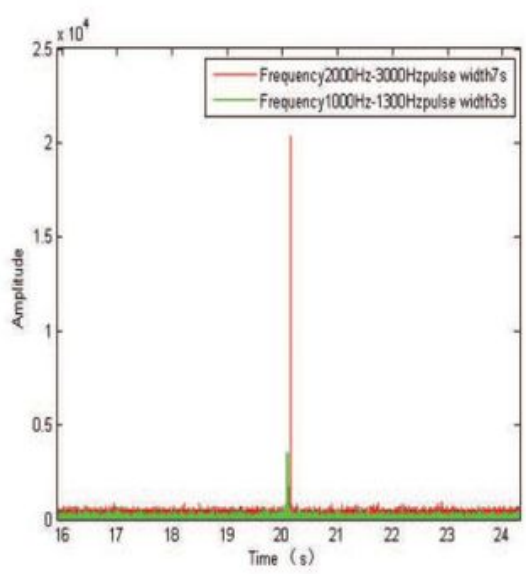

(c) $v=5 \mathrm{~m} / \mathrm{s}$.

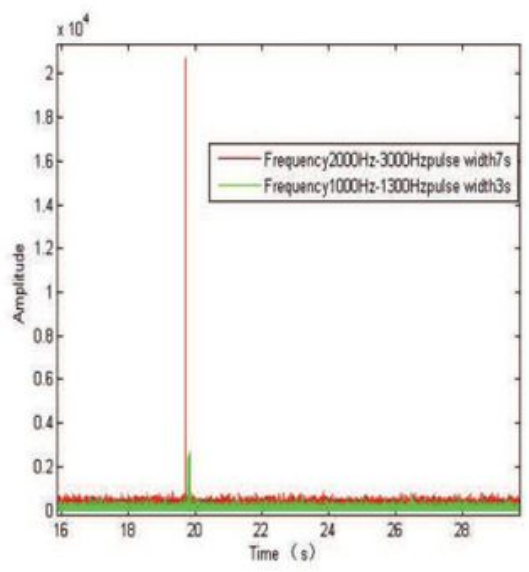

(e) $v=-10 \mathrm{~m} / \mathrm{s}$.

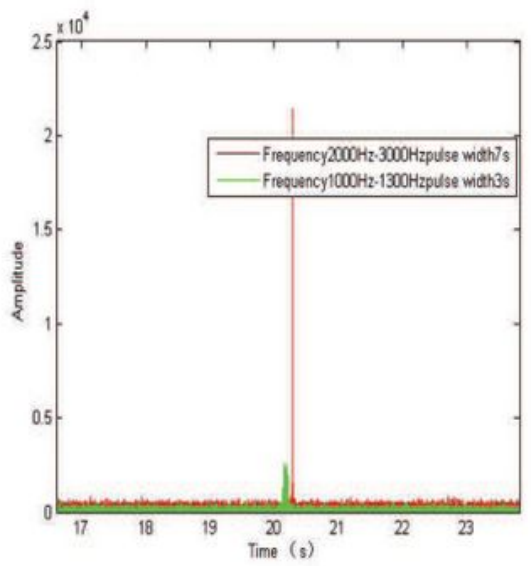

(b) $v=10 \mathrm{~m} / \mathrm{s}$.

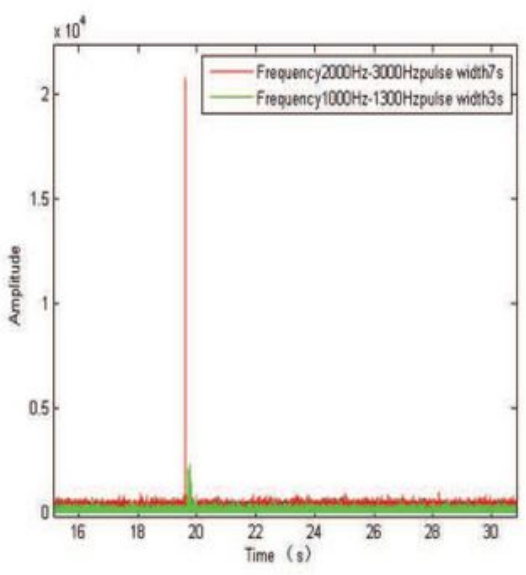

(d) $v=-15 \mathrm{~m} / \mathrm{s}$.

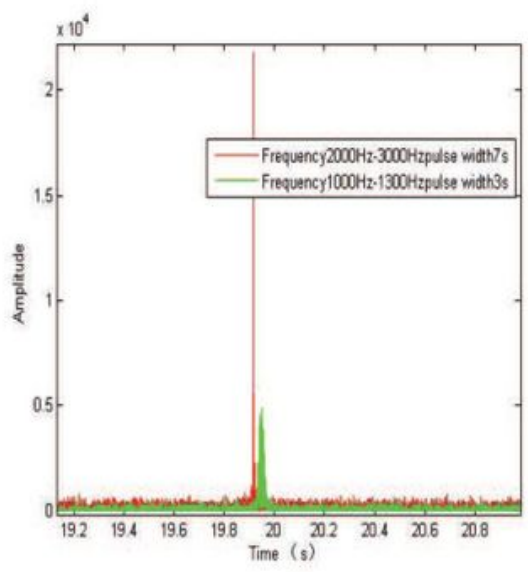

(f) $v=-3 m / s$.

\section{Figure 11}

Please see the Manuscript PDF file for the complete figure caption 


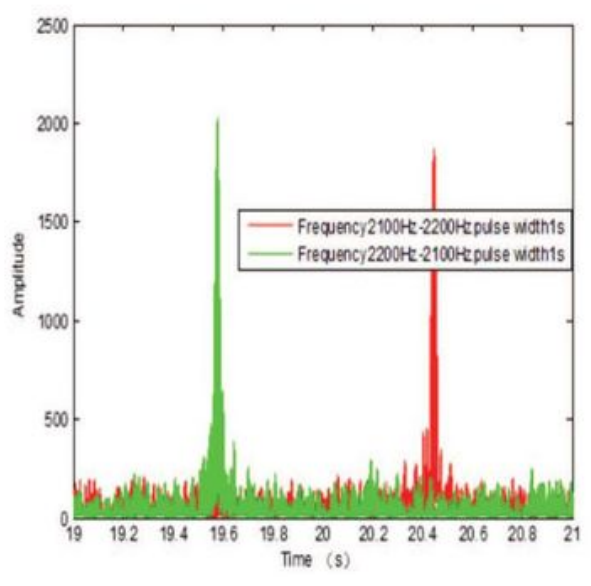

(a) $v=15 \mathrm{~m} / \mathrm{s}$.

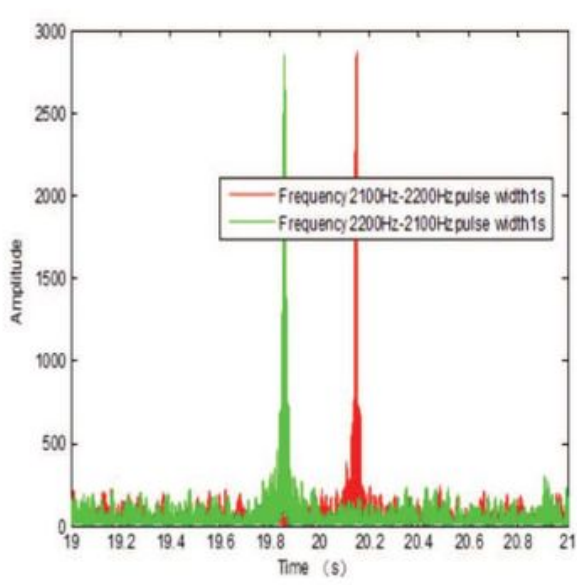

(c) $v=5 \mathrm{~m} / \mathrm{s}$.

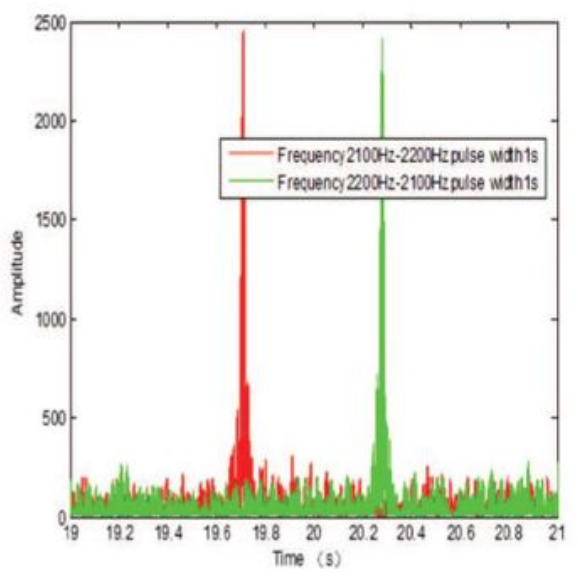

(e) $v=-10 \mathrm{~m} / \mathrm{s}$.

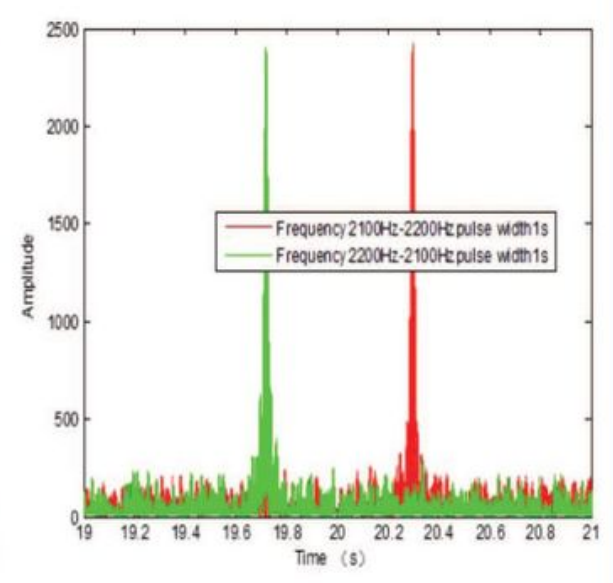

(b) $v=10 \mathrm{~m} / \mathrm{s}$.

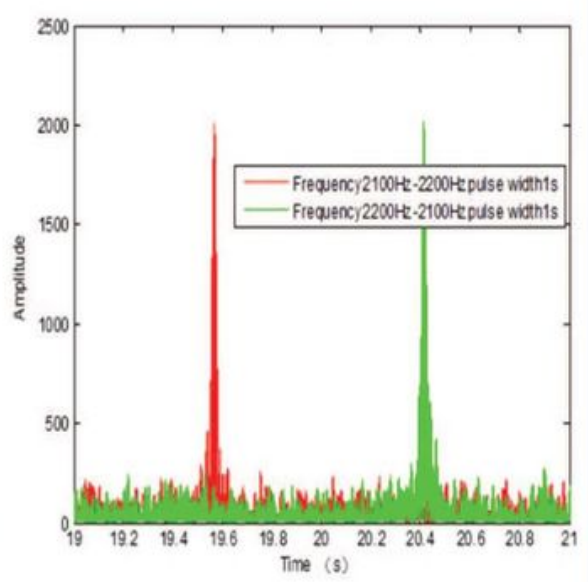

(d) $v=-15 \mathrm{~m} / \mathrm{s}$.

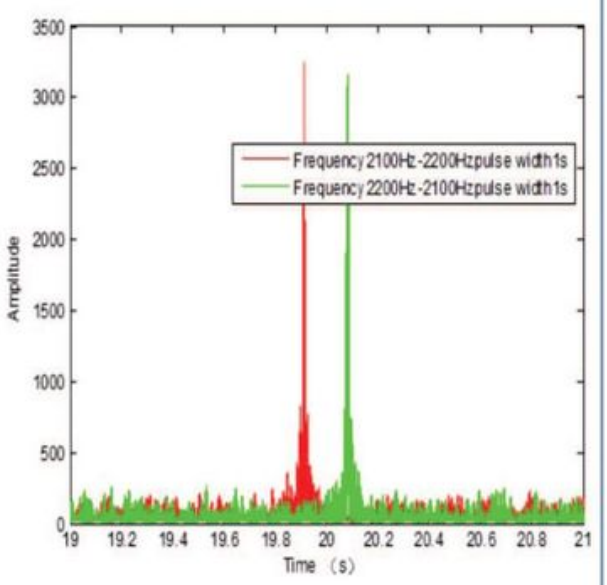

(f) $v=-3 m / s$.

\section{Figure 12}

Please see the Manuscript PDF file for the complete figure caption 